\title{
STOCHASTIC INTEGRATION WITH RESPECT TO FRACTIONAL BROWNIAN MOTION
}

\author{
Philippe CARMONA ${ }^{\mathrm{a}}$, Laure COUTIN ${ }^{\mathrm{a}}$, Gérard MONTSENY ${ }^{\mathrm{b}}$ \\ ${ }^{a}$ Laboratoire de statistique et probabilités, Université Paul Sabatier, 118, route de Narbonne, \\ 31062 Toulouse cedex 4, France \\ ${ }^{\mathrm{b}}$ L.A.A.S, 7, avenue du Colonel Roche, 31077 Toulouse cedex 4, France
}

Received 21 April 2000, revised 28 November 2001

Abstract. - For every value of the Hurst index $H \in(0,1)$ we define a stochastic integral with respect to fractional Brownian motion of index $H$. We do so by approximating fractional Brownian motion by semi-martingales.

Then, for $H>1 / 6$, we establish an Itô's change of variables formula, which is more precise than Privault's Ito formula (1998) (established for every $H>0$ ), since it only involves anticipating integrals with respect to a driving Brownian motion.

() 2003 Éditions scientifiques et médicales Elsevier SAS

MSC: primary 60G15, 60H07, 60H05; secondary 60J65, 60F25

Keywords: Gaussian processes; Stochastic integrals; Malliavin calculus; Fractional integration

RÉSUMÉ. - Pour tout $H \in(0,1)$, nous construisons une intégrale stochastique par rapport au mouvement Brownien fractionnaire de paramètre de Hurst $H$. Cette intégrale est basée sur l'approximation du mouvement Brownien fractionnaire par une suite de semi-martingales.

Ensuite, pour $H>1 / 6$, nous établissons une formule d'Itô qui précise celle obtenue par Privault (1998), au sens où elle ne comporte que des intégrales anticipantes par rapport à un mouvement Brownien directeur.

ㄷ 2003 Éditions scientifiques et médicales Elsevier SAS

\section{Introduction}

Fractional Brownian motion was originally defined and studied by Kolmogorov [14] within a Hilbert space framework. Fractional Brownian motion of Hurst index $H \in(0,1)$ is a centered Gaussian process $W^{H}$ with covariance

$$
\mathbb{E}\left[W_{t}^{H} W_{s}^{H}\right]=\frac{1}{2}\left(t^{2 H}+s^{2 H}-|t-s|^{2 H}\right) \quad(s, t \geqslant 0)
$$

E-mail addresses: carmona@cict.fr (P. Carmona), coutin@cict.fr (L. Coutin), montseny@laas.fr (G. Montseny). 
(for $H=\frac{1}{2}$ we obtain standard Brownian motion). Fractional Brownian motion has stationary increments

$$
\mathbb{E}\left[\left(W^{H}(t)-W^{H}(s)\right)^{2}\right]=|t-s|^{2 H} \quad(s, t \geqslant 0),
$$

and is $H$-self similar

$$
\left(\frac{1}{c^{H}} W^{H}(c t) ; t \geqslant 0\right) \stackrel{d}{=}\left(W^{H}(t) ; t \geqslant 0\right) \quad(\text { for all } c>0) .
$$

The Hurst parameter $H$ accounts not only for the sign of the correlation of the increments, but also for the regularity of the sample paths. Indeed, for $H>\frac{1}{2}$, the increments are positively correlated, and for $H<\frac{1}{2}$ they are negatively correlated. Furthermore, for every $\beta \in(0, H)$, its sample paths are almost surely Hölder continuous with index $\beta$. Finally, it is worthy of note that for $H>\frac{1}{2}$, according to Beran's definition [3], it is a long memory process: the covariance of increments at distance $u$ decrease as $u^{2 H-2}$.

These significant properties make fractional Brownian motion a natural candidate as a model of noise in mathematical finance (see Comte and Renault [5], Rogers [26]), and in communication networks (see, for instance, Leland, Taqqu and Willinger [16]).

Recently, there has been numerous attempts at defining a stochastic integral with respect to fractional Brownian motion. Indeed, for $H \neq \frac{1}{2} W^{H}$ is not a semi-martingale (see, e.g., Example 2 of Section 4.9.13 of Liptser and Shyriaev [19]), and usual Itô's stochastic calculus may not be applied. However, the integral

$$
\int_{0}^{t} a(s) \mathrm{d} W^{H}(s)
$$

may be defined for suitable $a$. On the one hand, since $W^{H}$ has almost its sample paths Hölder continuousof index $\beta$, for any $\beta<H$, the integral (1.1) exists in the RiemannStieljes sense (path by path) if almost every sample path of $a$ has finite $p$-variation with $\frac{1}{p}+\beta>1$ (see Young [32]): this is the approach used by Dai and Heyde [8] and Lin [18] when $H>\frac{1}{2}$. Let us recall that the $p$-variation of a function $f$ over an interval $[0, t]$ is the least upper bound of sums $\sum_{i}\left|f\left(x_{i}\right)-f\left(x_{i-1}\right)\right|^{p}$ over all partitions $0=x_{0}<x_{1}<\cdots<x_{n}=T$. A recent survey of the important properties of RiemannStieltjes integral is the concentrated advanced course of Dudley and Norvaisa [11]. An extension of Riemann-Stieltjes integral has been defined by Zähle [33], see also the recent work of Ruzmaikina [29], by means of composition formulas, integration by parts formula, Weyl derivative formula concerning fractional integration/differentiation, and the generalized quadratic variation of Russo and Vallois [27,28].

On the other hand, $W^{H}$ is a Gaussian process, and (1.1) can be defined for deterministic processes $a$ by way of an $L^{2}$ isometry: see, for example, Norros, Valkeila and Virtamo [21] or Pipiras and Taqqu [23]. With the help of stochastic calculus of variations (see [22]) this integral may be extended to random processes $a$. In this case, the stochastic integral (1.1) is a divergence operator, that is the adjoint of a stochastic 
gradient operator (see the pioneering paper of Decreusefond and Ustunel [9]). It must be noted that Duncan, Hu and Pasik-Duncan [12] have defined the stochastic integral in a similar way by using Wick product. Feyel and de La Pradelle [13], Ciesielski, Kerkyacharian and Roynette [4] also used the Gaussian property of $W^{H}$ to prove that $W^{H}$ belongs to suitable function spaces and construct a stochastic integral.

Eventually, Alos, Mazet and Nualart [1] have established, following the ideas introduced in a previous version of this paper, very sharp sufficient conditions that ensures existence of the stochastic integral (1.1).

The construction of the stochastic integral. The starting point of our approach is the following representation of fractional Brownian motion given by Decreusefond and Ustunel [9]

$$
W^{H}(t)=\int_{0}^{t} K^{H}(t, s) \mathrm{d} B(s),
$$

where

$$
K^{H}(t, s)=\frac{(t-s)^{H-1 / 2}}{\Gamma\left(H+\frac{1}{2}\right)} F\left(H-\frac{1}{2}, \frac{1}{2}-H, H+\frac{1}{2}, 1-\frac{t}{s}\right) \quad(s<t),
$$

where $F$ denotes Gauss hypergeometric function and $(B(t) ; t \geqslant 0)$ is a standard Brownian motion.

The first step we take is to define the integral of deterministic functions (Section 4). Observing that $K^{H}(t, s)=I_{t}^{H} 1_{[0, t]}(s)$ where $I_{t}^{H}$ is the integral operator

$$
I_{t}^{H} f(s)=K^{H}(t, s) f(s)+\int_{s}^{t}(f(u)-f(s)) \partial_{1} K^{H}(u, s) \mathrm{d} u,
$$

we define

$$
\int_{0}^{t} f(s) \mathrm{d} W^{H}(s)=\int_{0}^{t} I_{t}^{H} f(u) \mathrm{d} B_{u}
$$

for suitable deterministic functions.

In order to extend formula (1.4) to random processes $f$, we introduce the key idea of this paper, which is to approximate $W^{H}$ by processes of the type

$$
W_{K}(t)=\int_{0}^{t} K(t, s) \mathrm{d} B(s) \quad(t \in[0, T])
$$

with a kernel $K$ smooth enough to ensure that $W_{K}$ is a semi-martingale (Proposition 2.5). 
Then, for a such a semi-martingale kernel $K$ and a good integrand $a$, we prove the following generalization of (1.4)

$$
\int_{0}^{t} a(s) \mathrm{d} W_{K}(s)=\int_{0}^{t} I_{t} a(s) \mathrm{d} B(s)+\int_{0}^{t} I_{t} D_{s} a(s) \mathrm{d} s,
$$

where $D_{s} a$ denotes the stochastic gradient and $I_{t}$ is defined as $I_{t}^{H}$.

In Sections 2 and 3 topologies are defined on the space of kernels and the space of integrands. These are not 'natural' topologies but ad hoc ones, whose sole aim is to fulfill the following requirements:

(1) The mapping $(a, K) \rightarrow \int_{0}^{t} a(s) \mathrm{d} W_{K}(s)$ is bilinear continuous (Propositions 6.1).

(2) Rough kernels such as $K^{H}$ are in the closure of the space of semi-martingale kernels.

(3) For nice integrands $a$ the process $t \rightarrow \int_{0}^{t} a(s) \mathrm{d} W_{K}(s)$ has a continuous modification (Theorem 7.1).

Eventually, we are able to take limits in the Itô's formula established for semimartingale kernels in Proposition 8.1. Let $C_{b}^{n}$ be the set of functions $f$ whose derivatives, up to the order $n \in \mathbb{N}$, are continuous and bounded. Privault proved that for $f \in C_{b}^{2}$,

$$
\begin{aligned}
f\left(W^{H}(t)\right)= & f(0)+\int_{0}^{t} f^{\prime}\left(W^{H}(s)\right) \mathrm{d} W^{H}(s) \\
& +\frac{1}{2} \int_{0}^{t} f^{\prime \prime}\left(W^{H}(s)\right)\left(c_{H} 2 H s^{2 H-1}\right) \mathrm{d} s,
\end{aligned}
$$

where $\int_{0}^{t} f^{\prime}\left(W^{H}(s)\right) \mathrm{d} W^{H}(s)$ is the $L^{2}$-limit of divergences (Skorokhod integrals). For $H>1 / 4$ and $f \in C_{b}^{3}$, we show that this integral, which is not in general the limit of Riemann sums, is a Skorokhod integral with respect to the driving Brownian motion:

$$
\begin{aligned}
f\left(W^{H}(t)\right)= & f(0)+\int_{0}^{t} I_{t}^{H} f^{\prime}\left(W^{H}\right)(s) \mathrm{d} B_{s} \\
& +\frac{1}{2} \int_{0}^{t} f^{\prime \prime}\left(W^{H}(s)\right)\left(c_{H} 2 H s^{2 H-1}\right) \mathrm{d} s .
\end{aligned}
$$

By the same procedure, we have been able to prove an Itô's formula for $H>1 / 6$, in Proposition 8.11. We do not give here this formula since it is complex (5 lines) and does not seem to be an easy starting point for a generalization to every $H \in(0,1)$.

To end this rather lengthy introduction we try to give an answer to a question that nearly everyone involved with stochastic integration with respect to Fractional Brownian motion has asked us.

"What is the difference between the stochastic approach and the pathwise approach to integration with respect to Fractional Brownian motion?" 
On the one hand we prove in the Appendix that for $W_{1}^{H}, W_{2}^{H}$ two independent fractional Brownian motions of index $1 / 4<H<1 / 2$, the integral

$$
\int_{0}^{t} W_{1}^{H}(s) \mathrm{d} W_{2}^{H}(s)
$$

cannot be defined for the classical and generalized pathwise integrals. On the other hand, a slight generalization of our results enabled Coutin and Qian [7,6] to show that for $\frac{1}{4}<H<\frac{1}{2}$ the integral $\int_{0}^{t} W_{1}^{H}(s) \mathrm{d} W_{2}^{H}(s)$ can be defined as a process with a continuous modification.

Our main source of inspiration for Itô's formula is T. Lyons paper [20], and in particular the idea that in order to integrate with respect to rough signals, you may sometimes have to replace approximating Riemann sums, of order 1, with Taylor sums of higher order. More precisely, you may want to replace $f\left(W^{H}\left(t_{i+1}\right)\right)-f\left(W^{H}\left(t_{i}\right)\right)$ by

$$
f\left(W^{H}\left(t_{i+1}\right)\right)-f\left(W^{H}\left(t_{i}\right)\right)-\left(W^{H}\left(t_{i+1}\right)-W^{H}\left(t_{i}\right)\right) f^{\prime}\left(W^{H}\left(t_{i}\right)\right)+\cdots .
$$

\section{The vector spaces of kernels}

The spaces of integrands and kernels we shall work with depend on parameters $(p, \gamma, \theta)$. We shall assume from now on that the set $(p, \gamma, \theta)$ of parameters is admissible, that is

$$
\begin{aligned}
& 1<p<2, \quad \frac{1}{p}+\frac{1}{q}=1, \quad \frac{2}{q}>\gamma>\frac{1}{q}, \quad \delta=\frac{2 p}{2-p} \\
& h_{\gamma}=\frac{1}{\theta^{*}} \leqslant \frac{2}{q}-\gamma, \quad \frac{1}{\theta / 2}+\frac{1}{\theta^{*} / 2}=1 .
\end{aligned}
$$

Observe that $\delta / 2$ and $q / 2$ are conjugated Hölder exponents, and that $1>2 / q$.

Let $K: \mathbb{R}^{2} \rightarrow \mathbb{R}$ be a measurable kernel such that

$$
\begin{array}{ll}
\int_{0}^{t} K(t, s)^{2} \mathrm{~d} s<+\infty & \text { for every } t>0, \\
K(t, s)=0 & \text { if } s>t .
\end{array}
$$

We consider the adapted Gaussian process

$$
W_{K}(t)=\int_{0}^{t} K(t, s) \mathrm{d} B_{s},
$$

where $\left(B_{t} ; t \geqslant 0\right)$ is a standard Brownian motion.

DEFINITION 2.1. - We say that $K$ is a rough kernel if there exists a measurable function $(t, s) \rightarrow \partial_{1} K(t, s)$, such that $u \rightarrow \partial_{1} K(u, s)$ is integrable on every $\left[t, t^{\prime}\right] \subset$ 
$(s,+\infty)$ and satisfies

$$
K\left(t^{\prime}, s\right)-K(t, s)=\int_{t}^{t^{\prime}} \partial_{1} K(u, s) \mathrm{d} u \quad\left(s<t<t^{\prime}\right) .
$$

We say that $K$ is a smooth kernel if $K$ is a rough kernel that satisfies

$$
K(t, s)-K(s, s)=\int_{s}^{t} \partial_{1} K(u, s) \mathrm{d} u \quad(s<t) .
$$

The topology on the space of rough kernels is defined via mixed Lebesgue spaces (see Stroock [31], Section 6.2). Let $\left(E_{1}, \mathcal{B}_{1}, \mu_{1}\right)$ and $\left(E_{2}, \mathcal{B}_{2}, \mu_{2}\right)$ be a pair of $\sigma$ finite measure spaces and let $p_{1}, p_{2} \in[1, \infty)$. Given a measurable function $f$ on $\left(E_{1} \times E_{2}, \mathcal{B}_{1} \times \mathcal{B}_{2}\right)$, define

$$
\|f\|_{p_{1}, p_{2}}=\left[\int_{E_{2}}\left(\int_{E_{1}}\left|f\left(x_{1}, x_{2}\right)\right|^{p_{1}} \mu_{1}\left(\mathrm{~d} x_{1}\right)\right)^{p_{2} / p_{1}} \mu_{2}\left(\mathrm{~d} x_{2}\right)\right]^{1 / p_{2}},
$$

and let $L^{\left(p_{1}, p_{2}\right)}\left(\mu_{1}, \mu_{2}\right)$ denote the mixed Lebesgue space of $\mathbb{R}$ valued, $\mathcal{B}_{1} \times \mathcal{B}_{2}$ measurable $f$ for which $\|f\|_{p_{1}, p_{2}}<\infty$.

For $i=1,2$ we let $\left(E_{i}, \mathcal{B}_{i}, \mu_{i}\right)=((0, t), \mathcal{B}(0, t), \mathrm{d} s)$ be the space $(0, t)$ endowed with the $\sigma$-field of Borel sets and Lebesgue measure, $p_{1}=p$ and $p_{2}=\delta, f(u, s)=$ $\partial_{1} K(u, s)(u-s)^{\gamma} 1_{(0<s<u)}$ and $\|K\|_{\gamma, \theta, p, t} \stackrel{\text { def }}{=}\|f\|_{p, \delta}+\|K(t, \cdot)\|_{\delta}$, that is

$$
\|K\|_{\gamma, \theta, p, t} \stackrel{\text { def }}{=}\left(\int_{0}^{t}\left(\int_{s}^{t}\left|\partial_{1} K(u, s)(u-s)^{\gamma}\right|^{p} \mathrm{~d} u\right)^{\delta / p} \mathrm{~d} s\right)^{1 / \delta}+\left(\int_{0}^{t}|K(t, s)|^{\theta} \mathrm{d} s\right)^{1 / \theta} .
$$

Let $E_{\gamma, \theta, p, t}$ be the space of rough kernels $K$ such that $\|K\|_{\gamma, \theta, p, t}<+\infty$.

LEMMA 2.2. $-E_{\gamma, \theta, p, t}$ is a Banach space.

Proof. - Assume that $\left(K_{n}\right)_{n \in \mathbb{N}}$ is a Cauchy sequence in $E_{\gamma, \theta, p, t}$. Then the sequence of functions $f_{n}(u, s)=\partial_{1} K_{n}(u, s)(u-s)^{\gamma} 1_{(0<s<u)}$ is a Cauchy sequence in $L^{(p, 2 p / 2-p)}$. Therefore it converges in $L^{(p, 2 p / 2-p)}$ to a function $f$ and we let $z(u, s)=f(u, s)(u-$ $s)^{-\gamma}$. Since $\left(K_{n}(t, \cdot)\right)_{n \in \mathbb{N}}$ is a Cauchy sequence in $L^{\theta}(0, t)$ it converges in $L^{\theta}$ to a function $\phi \in L^{\theta}(0, t)$. Finally, letting $K$ be the kernel

$$
K(v, s)= \begin{cases}\phi(s)-\int_{v}^{t} z(u, s) \mathrm{d} u & \text { if } 0<s<v<t \\ 0 & \text { if } v \leqslant s \leqslant t\end{cases}
$$

we conclude that $K$ is a rough kernel for which $\left\|K-K_{n}\right\|_{\gamma, \theta, p, t} \rightarrow 0$ since by construction:

$$
\left\|K-K_{n}\right\|_{\gamma, \theta, p, t}=\left\|f-f_{n}\right\|_{p, \delta}+\left\|K_{n}(t, \cdot)-\phi(\cdot)\right\|_{\theta} .
$$


Remark 2.3. - It is quite straightforward to prove, via Hölder's inequality, that for $t \leqslant T$, the space $E_{\gamma, \theta, p, T}$ can be continuously embedded in $E_{\gamma, \theta, p, t}$. Indeed, we only need to prove that $\|K(T, \cdot)-K(t, \cdot)\|_{L^{\theta}(0, t)}<+\infty$. We first observe that fors $<t$ :

$$
\begin{aligned}
|K(T, s)-K(t, s)| & =\left|\int_{t}^{T} \partial_{1} K(u, s) \mathrm{d} u\right| \\
& \leqslant(t-s)^{1 / q-\gamma}\left(\int_{t}^{T}\left|\partial_{1} K(u, s)(u-s)^{\gamma}\right|^{p} \mathrm{~d} u\right)^{1 / p} .
\end{aligned}
$$

Therefore, applying Hölder's inequality to the pair of conjugated exponents $(r=$ $\left.\delta / \theta, r^{\prime}\right)$

$$
\int_{0}^{t}|K(T, s)-K(t, s)|^{\theta} \mathrm{d} s \leqslant C\left(\int_{0}^{t}(t-s)^{\theta(1 / q-\gamma) r^{\prime}} \mathrm{d} s\right)^{1 / r^{\prime}}\|K\|_{\gamma, \theta, p, T}^{\theta} .
$$

The first factor may be majorized by a constant $C$, since $\frac{2}{q}-\gamma>\frac{1}{\theta^{*}}$ implies $\theta\left(\frac{1}{q}-\gamma\right) r^{\prime}>$ -1 .

Let us recall that our main results are proved by approximating the fractional Brownian motion kernel by smooth kernels for which $W_{K}$ is a semi-martingale.

DEFINITION 2.4. - We say that the smooth kernel $K$ is a semi-martingale kernel if

$$
\sup _{u \leqslant t}\left(\int_{0}^{u} \partial_{1} K(u, s)^{2} \mathrm{~d} s\right)+\int_{0}^{t} K(s, s)^{2} \mathrm{~d} s<+\infty \quad(\forall t>0) .
$$

PROPOSITION 2.5. -

(1) If $K$ is a semi-martingale kernel, then $W_{K}$ is a semi-martingale with decomposition

$$
W_{K}(t)=\int_{0}^{t} K(s, s) \mathrm{d} B_{s}+\int_{0}^{t} W_{\partial_{1} K}(s) \mathrm{d} s .
$$

(2) The vector space $F_{\gamma, \theta, p, t}$ of semi-martingale kernels in $E_{\gamma, \theta, p, t}$ is dense in $E_{\gamma, \theta, p, t}$.

Proof. - (1) The definition of a smooth kernel is more than what we need to apply Fubini's Stochastic Theorem (see, e.g., Protter [25], Theorem 46):

$$
\begin{aligned}
W_{K}(t) & =\int_{0}^{t}\left(K(s, s)+\int_{s}^{t} \partial_{1} K(u, s) \mathrm{d} u\right) \mathrm{d} B_{s} \\
& =\int_{0}^{t} K(s, s) \mathrm{d} B_{s}+\int_{0}^{t}\left(\int_{0}^{u} \partial_{1} K(u, s) \mathrm{d} B_{s}\right) \mathrm{d} u .
\end{aligned}
$$


(2) Let $\varepsilon>0$ be given. We perform the change of variables $u=v+s$ in the definition of $\|K\|_{\gamma, \theta, p, t}$, and we let $g(v, s)=\partial_{1} K(v+s, s) v^{\gamma} 1_{(0<v<t-s)}$. Then $g$ is in $L^{(p, \delta)}((0, t) \times(0, t))$ and by Lemma 6.2.11 of Stroock [31] can be approached at distance less than $\varepsilon$ by a function $h(v, s)$ having the form

$$
h(v, s)=\sum_{i=1}^{n} \phi_{i}(v) \psi_{j}(s)
$$

where the $\psi_{i}, \phi_{i}$ are in $L^{\infty}(0, t)$ and the $\phi_{i}$ have disjoint support. Therefore, if we let

$$
\Lambda(r, s)=K(t, s)-\int_{r}^{t} h(u-s, s)(u-s)^{-\gamma} \mathrm{d} u \quad(s<r<t),
$$

then $\Lambda$ is a kernel such that $\|K-\Lambda\|_{\gamma, \theta, p, t} \leqslant \varepsilon$. Furthermore since $\partial_{1} \Lambda(r, s)=h(r-$ $s, s)(r-s)^{-\gamma}$ for $0<s<r<t$, and $h$ is bounded, we see that $\Lambda$ is a smooth kernel. However, $\Lambda$ is not a semi-martingale kernel if $\gamma>\frac{1}{2}$. Hence for $\varepsilon>0$ we consider

$$
\Lambda_{\varepsilon}(r, s)=K(t, s)-\int_{r}^{t} h(u-s, s)(u+\varepsilon-s)^{-\gamma} \mathrm{d} u \quad(s<r<t) .
$$

The $\Lambda_{\varepsilon}$ is a semi-martingale kernel, and

$$
\left\|\Lambda-\Lambda_{\varepsilon}\right\|_{\gamma, \theta, p, t}^{\delta} \leqslant \text { (const) } \int_{0}^{t} \mathrm{~d} s\left(\int_{s}^{t}\left|\left(\frac{u-s}{u+\varepsilon-s}\right)^{\gamma}-1\right| \mathrm{d} u\right)^{\delta / p} \rightarrow 0
$$

as $\varepsilon \rightarrow 0$ by dominated convergence.

DEFINITION 2.6. - In order to prove the continuity of our stochastic integral we introduce $A_{\gamma, \theta, p, T}^{\beta}$ the subset of $E_{\gamma, \theta, p, T}$ of kernels $K$ such that

$$
\|K\|_{\beta}^{2}=\sup _{0<t<t+\tau \leqslant T} \tau^{-\left(h_{\gamma}+\beta\right)} \int_{0}^{t}\left[\int_{t}^{t+\tau}(u-s)^{\beta} \partial_{1} K(u, s) \mathrm{d} u\right]^{2} \mathrm{~d} s<+\infty
$$

and endow it with the norm

$$
\||K|\|=\|K\|_{\gamma, \theta, p, T}+\|K\|_{\beta} .
$$

Since Itô's formula is proved by approximating with smooth kernels, we introduce $B_{\gamma, \theta, p, T}^{\beta}$ the closure of $F_{\gamma, \theta, p, T}$ in $A_{\gamma, \theta, p, T}^{\beta}$.

\subsection{The case of fractional Brownian motion}

We recall here the basic properties of hypergeometric functions required throughout this paper (see, e.g., Chapter 9 of Lebedev [15]). Gauss hypergeometric function 
$F(\alpha, \beta, \gamma, z)$ is defined for every $\alpha, \beta, \gamma,|z|<1$ and $\gamma \neq 0,-1, \ldots$ by

$$
F(\alpha, \beta, \gamma, z)=\sum_{k \geqslant 0} \frac{(\alpha)_{k}(\beta)_{k}}{(\gamma)_{k} k !} z^{k},
$$

where $(\alpha)_{0}=1$ and $(\alpha)_{k}=\Gamma(\alpha+k) / \Gamma(\alpha)=\alpha(\alpha+1) \cdots(\alpha+k-1)$ is the Pochammer index. The convergence radius of this series is 1 and, as soon as $\operatorname{Re}(\gamma-\beta-\alpha)>0$, $\lim _{z \rightarrow 1} F(\alpha, \beta, \gamma, z)$ exists and is finite. Obviously we have

$$
F(\alpha, \beta, \gamma, z)=F(\beta, \alpha, \gamma, z), \quad F(\alpha, \beta, \gamma, 0)=1 .
$$

Furthermore, if $|\arg (1-z)|<\pi$ and $\operatorname{Re}(\gamma)>\operatorname{Re}(\beta)>0$,

$$
F(\alpha, \beta, \gamma, z)=\frac{\Gamma(\gamma)}{\Gamma(\beta) \Gamma(\gamma-\beta)} \int_{0}^{1} u^{\beta-1}(1-u)^{\gamma-\beta-1}(1-z u)^{-\alpha} \mathrm{d} u .
$$

The hypergeometric function $F\left(\alpha^{\prime}, \beta^{\prime}, \gamma^{\prime}, \cdot\right)$ is said to be contiguous to $F(\alpha, \beta, \gamma, \cdot)$ if $\left|\alpha-\alpha^{\prime}\right|=1$ or $\left|\beta-\beta^{\prime}\right|=1$ or $\left|\gamma-\gamma^{\prime}\right|=1$. If $F_{1}$ and $F_{2}$ are hypergeometric functions contiguous to $F$, then there exists a relation of the type

$$
P(z) F(z)+P_{1}(z) F_{1}(z)+P_{2}(z) F_{2}(z)=0 \quad(|\arg (1-z)|<\pi),
$$

with $P(z), P_{1}(z), P_{2}(z)$ polynomials in the variable $z$. These relations ensure that there exists an analytical continuation of $F(\alpha, \beta, \gamma, z)$ to the domain

$$
\mathbb{C} \times \mathbb{C} \times(\mathbb{C} \backslash\{0,-1,-2, \ldots\}) \times\{z:|\arg (1-z)|<\pi\} .
$$

We shall also use the asymptotic estimate, for $|\arg (1-z)|<\pi, \operatorname{Re}(\Gamma)>\operatorname{Re}(\alpha)>$ $0, \operatorname{Re}(\beta)<0$ :

$$
F(\alpha, \beta, \gamma, z) \sim \frac{\Gamma(\gamma) \Gamma(\alpha-\beta)}{\Gamma(\alpha) \Gamma(\gamma-\beta)}(-z)^{-\beta} \quad(|z| \rightarrow \infty) .
$$

LEMMA 2.7. - Given $s>0$ and $H \neq \frac{1}{2}$, the function $t \rightarrow K^{H}(t, s)$ is differentiable on $(s,+\infty)$ with derivative

$$
\partial_{1} K^{H}(t, s)=\frac{(s / t)^{1 / 2-H}}{\Gamma\left(H-\frac{1}{2}\right)}(t-s)^{H-3 / 2} \quad(0<s<t) .
$$

Furthermore, $K^{H} \in A_{\gamma, \theta, p, T}^{\beta}$ for admissible parameters $(p, q, \gamma, \ldots)$ such that

$$
\frac{1}{p}+\gamma>3 / 2-H, \quad-\theta\left|H-\frac{1}{2}\right|+1>0 \quad \text { and } \quad \beta+H<1 .
$$

In particular, for $0<\varepsilon<H<\frac{1}{2}$, there exists a set of admissible parameters for which $h_{\gamma}=H-\varepsilon$. 
Remark 2.8. - Observe that for $H<\frac{1}{2}$, we have $\gamma-\frac{1}{q}>1 / 2-H>0$.

Proof. - Let us state three facts:

(1) From the analyticity of the Gauss function, we deduce that the function $t \rightarrow$ $K^{H}(t, s)$ is differentiable on $(s,+\infty)$ with a derivative $f(H, t, s)$ such that $H \rightarrow f(H, t, s)$ is holomorphic on $\left\{H \in \mathbb{C}: \operatorname{Re}(H)>-\frac{1}{2}\right\}$.

(2) The integral representation (2.10) implies that for $H \in(1 / 2,1)$ the function $t \rightarrow K^{H}(t, s)$ is differentiable on $(s,+\infty)$ with derivative $\partial_{1} K^{H}(t, s)$.

(3) The function $H \rightarrow \partial_{1} K^{H}(t, s)$ is holomorphic in the region

$$
\Delta=\{H \in \mathbb{C}: 0<\operatorname{Re}(H)<1\} \backslash\left\{\frac{1}{2}\right\} .
$$

Therefore, by analytic continuation: for $0<s<t, \partial_{1} K^{H}(t, s)=f(H, t, s)$ on $\Delta$.

To determine the admissible set of parameters for which $K^{H} \in E_{\gamma, \theta, p, T}$, we just use the fact that $(s, t) \rightarrow K^{H}(t, s)$ is continuous on $\{0<s<t\}$, combined with the following consequence of the asymptotic estimate of $F$,

$$
\begin{aligned}
& K^{H}(t, s) \sim_{s \rightarrow 0+}(\text { const }) s^{-|H-1 / 2|} \\
& K^{H}(t, s) \sim_{s \rightarrow t-}(\text { const })(t-s)^{H-1 / 2} .
\end{aligned}
$$

Therefore, given $0<\varepsilon<H<\frac{1}{2}$, we can set $\frac{1}{q}=\frac{1}{2}-\frac{\varepsilon}{3}$ and $h_{\gamma}=\frac{1}{\theta^{*}}=H-\varepsilon$, $\frac{2}{q}-\gamma=H-\varepsilon / 2$ to get $q>2,2 / q>\gamma$, and $\gamma-\frac{1}{q}=\frac{1}{q}-(H-\varepsilon / 2)>\frac{1}{2}-H>0$.

It remains to show that $\|K\|_{\beta}<+\infty$ (indeed, we can then approximate $K$ by the sequence $K_{n}(t, s)=K\left(t+\frac{1}{n}, s\right)$ of smooth kernels in $\left.F_{\gamma, \theta, p, T}\right)$ : for $0<t<t+\tau \leqslant T$,

$$
\begin{aligned}
& \int_{0}^{t}\left(\int_{t}^{t+\tau}(u-s)^{\beta} \partial_{1} K(u, s) \mathrm{d} u\right)^{2} \mathrm{~d} s \\
& \quad \leqslant \int_{0}^{t}(t-s)^{-1+2 \varepsilon}\left(\int_{t}^{t+\tau}(u-s)^{\beta+H-1-\varepsilon} \mathrm{d} u\right)^{2} \mathrm{~d} s \\
& \quad \leqslant C \tau^{2(\beta+H-\varepsilon)} .
\end{aligned}
$$

\subsection{Properties of integral operators associated to kernels}

Let $X$ be a separable Hilbert space with norm $|\cdot|_{X}$, and $K$ be a rough kernel. To a measurable $a:[0, T] \rightarrow X$ we associate

$$
I_{t}^{K}(a)(s)=K(t, s) a(s)+\int_{s}^{t}(a(u)-a(s)) \partial_{1} K(u, s) \mathrm{d} u,
$$

as soon as the integral in the right-hand side makes sense for almost every $s \in[0, T]$. 
Similarly we shall denote by $J_{t}^{K}$ the integral operator defined on measurable $f:[0, T]^{2} \rightarrow X$ by

$$
J_{t}^{K}(f)(s)=\int_{s}^{t} f(u, s) \partial_{1} K(u, s) \mathrm{d} u,
$$

as soon as the integral in the right-hand side makes sense for almost every $s \in[0, T]$.

Observe that if $\Delta_{s, u}^{0} a=a(u)-a(s)$, then

$$
I_{t}^{K}(a)(s)=K(t, s) a(s)+J_{t}^{K}\left(\Delta^{0} a\right)(s) .
$$

Since $K$ and $\partial_{1} K$ need not be positive, we introduce a dominating positive kernel

$$
K_{+}(t, s)=\left[|K(T, s)|+\int_{t}^{T}\left|\partial_{1} K(u, s)\right| \mathrm{d} u\right] 1_{(s<t)} .
$$

For $0 \leqslant s<t \leqslant t+\tau \leqslant T$ we have

$$
\begin{aligned}
& |K(t+\tau, s)-K(t, s)| \leqslant K_{+}(t, s)-K_{+}(t+\tau, s), \\
& |K(t, s)| \leqslant K_{+}(t, s) .
\end{aligned}
$$

For $\beta \in \mathbb{R}, \beta \neq 0$, we let $w_{\beta}$ be the weight

$$
w_{\beta}(u, s)=\max (u-s, 0)^{\beta} .
$$

Then if $\mathrm{d} u \mathrm{~d} s$ denotes the Lebesgue measure on $[0, T]^{2}$, we introduce the Lebesgue space

$$
L_{w_{\beta}}^{q}(X)=L^{q}\left([0, T]^{2} ; X ; w_{\beta}(u, s) \mathrm{d} u \mathrm{~d} s\right) .
$$

Eventually we denote by $\mathcal{H}^{\beta}(X)$ the set of $\beta$ Hölder continuous functions taking their values in $X$ :

$$
\mathcal{H}^{\beta}(X)=\left\{f:[0, T] \rightarrow X,\|f\|_{\mathcal{H}^{\beta}(X)}=\sup _{0 \leqslant u<s \leqslant T} \frac{|f(u)-f(s)|_{X}}{|u-s|^{\beta}}<+\infty\right\} .
$$

For functions of two variables $f(u, s)$ we introduce

$$
\tilde{\mathcal{H}}^{\beta}(X)=\left\{f:[0, T]^{2} \rightarrow X,\|f\|_{\tilde{\mathcal{H}}^{\beta}(X)}=\sup _{0 \leqslant u<s \leqslant T} \frac{|f(u, s)|_{X}}{|u-s|^{\beta}}<+\infty\right\} .
$$

Through the rest of this paper $(\gamma, \delta, p)$ denotes a set of admissible parameters, $T>0$ and $\beta \in \mathbb{R}^{*}$. The constant $C$ may vary from line to line but depends only on $(\gamma, \delta, p, T, \beta)$; to ease notation, we shall omit this dependency when the context allows it.

PROPOSITION 2.9. - For every fixed $t \in[0, T]$

(1) The mappings $J:(f, K) \rightarrow J_{\vdash}^{K}(f)(t)$ and $J^{+}:(f, K) \rightarrow J_{t}^{K_{+}}(f)$ are bilinear continuous from $L_{w_{-\gamma q}}^{q}(X) \times E_{\gamma, \theta, p, T}$ to $L^{2}\left([0, T]^{2} ; \mathrm{d} s, X\right)$. 
(2) The mapping $I_{t}:(a, K) \rightarrow I_{t}^{K}(a)$ is bilinear continuous from $L^{\theta^{*}}([0, T] ; \mathrm{d} s ; X) \cap$ $\left\{a: \Delta^{0} a \in L_{w_{-\gamma q}}^{q}(X)\right\} \times E_{\gamma, \theta, p, T}$ to $L^{2}\left([0, T]^{2} ; \mathrm{d} s, X\right)$.

Proof. - Since $\left|J_{t}^{K}(f)\right|_{X} \leqslant J_{t}^{K_{+}}\left(|f|_{X}\right)$, we can suppose, without any loss in generality, that $X=\mathbb{R}$ and study $J^{K_{+}}$.

First, apply Hölder's inequality to the pair of conjugated exponents $(p, q)$ :

$$
\left|J_{t}^{K_{+}}(f)(s)\right| \leqslant\left(\int_{s}^{t} w_{-\gamma q}(u, s)|f|^{q}(u, s) \mathrm{d} u\right)^{1 / q}\left(\int_{s}^{t} w_{\gamma p}(u, s)\left|\partial_{1} K(u, s)\right|^{p} \mathrm{~d} u\right)^{1 / p} .
$$

Then, apply Hölder's inequality to $(q / 2, \delta / 2)$ to get

$$
\left\|J_{t}^{K_{+}}(f)(\cdot)\right\|_{L^{2}([0, T], \mathrm{d} s)} \leqslant\|f\|_{L_{w_{-\gamma q}}^{q}(\mathbb{R})}\|K\|_{\gamma, \theta, p, T} .
$$

The second assertion is clearly a consequence of the first one, another Hölder's inequality for $\left(\theta / 2, \theta^{*} / 2\right)$, and Remark 2.3:

$$
\begin{aligned}
\|K(t, \cdot) a(\cdot)\|_{L^{2}([0, T], d s)} & \leqslant\|a\|_{L^{\theta^{*}([0, T] ; \mathbb{R})}}\|K\|_{\gamma, \theta, p, t} \\
& \leqslant C\|a\|_{L^{\theta^{*}([0, T] ; \mathbb{R})}\|K\|_{\gamma, \theta, p, T} .}
\end{aligned}
$$

The next proposition establish the Hölder regularity of a rough Kernel $K(t, s)$ with respect to the first variable $t$.

Proposition 2.10. - The space $E_{\gamma, \theta, p, T}$ may be continuously embedded into $\mathcal{H}^{h_{\gamma}}\left(L^{2}([0, T] ; \mathrm{d} s)\right)$.

Furthermore, the mapping $K \rightarrow K_{+}$is continuous from $E_{\gamma, \theta, p, T}$ into $\mathcal{H}^{h_{\gamma}}\left(L^{2}([0, T]\right.$; $\mathrm{d} s))$.

Proof. - According to the inequalities (2.13), we only need to prove the second point. The embedding is the following: we identify $K_{+}$with the function $F:[0, T] \rightarrow X=$ $L^{2}([0, T] ; \mathrm{d} s)$ defined by $F(t)=K_{+}(t, \cdot)$. Let $0 \leqslant t \leqslant t+\tau \leqslant T$; we have, for $s \neq t$ :

$$
\begin{aligned}
& K_{+}(t+\tau, s)-K_{+}(t, s)=|K(T, s)| 1_{(t, t+\tau)}(s)+J_{T}^{K_{+}}(f+g)(s) \\
& \text { with } \quad f(u, s)=1_{(t, t+\tau)}(s) 1_{(t+\tau, T)}(u), \quad g(u, s)=1_{(0, t)}(s) 1_{(t, t+\tau)}(u) .
\end{aligned}
$$

According to Lemma B.1, there exists a constant $C$ such that:

$$
\|f\|_{L_{w_{-\gamma q}}^{q}}+\|g\|_{L_{w_{-\gamma q}}^{q}} \leqslant C|\tau|^{2 / q-\gamma}
$$

and

$$
\left\|1_{(t, t+\tau)}\right\|_{L^{\theta^{*}([0, T], \mathrm{ds})}} \leqslant C \tau^{1 / \theta^{*}} .
$$

Therefore, since $h_{\gamma}=\frac{1}{\theta^{*}}<2 / q-\gamma$, we only need to apply Proposition 2.9 to $f, g, 1_{(t, t+\tau)}$ to obtain the continuity of $K \rightarrow K_{+}$.

Similarly, we can establish Hölder regularity for the operators $J$ and $J^{+}$.

PROPOSITION 2.11.- The operators $J$ and $J^{+}$are bilinear continuous from $\tilde{\mathcal{H}}^{\beta}(X) \times A_{\gamma, \theta, p, T}^{\beta}$ to $\mathcal{H}^{\beta+h}\left(L^{2}([0, T]) ; \mathrm{d} s ; X\right)$ as soon as $0<h<h_{\gamma}$. 
Proof. - Let $0 \leqslant t \leqslant t+\tau \leqslant T$ and $\tilde{K}$ be one of $K, K_{+}$. Then,

$$
\Delta_{t, t+\tau}^{0} J^{\tilde{K}}(a)(s)=J_{t+\tau}^{\tilde{K}}(a)(s)-J_{t}^{\tilde{K}}(a)(s)=J_{t+\tau}^{\tilde{K}}(a)(\max (s, t)) .
$$

We plug in the estimate $|a(u, s)| \leqslant\|a\|_{\tilde{\mathcal{H}}^{\beta}} w_{\beta}(u, s)$ for $u>s$, and get

$$
\left|\Delta_{t, t+\tau}^{0} J^{\tilde{K}}(a)(s)\right| \leqslant \Delta_{t, t+\tau}^{0} J^{\tilde{K}}\left(w_{\beta}\right)(s)\|a\|_{\tilde{\mathcal{H}}^{\beta}}
$$

and thus the proposition is an easy consequence of the following lemma.

LEMMA 2.12. - The mapping $K \rightarrow J^{\tilde{K}}\left(w_{\beta}\right)$ is linear continuous from $A_{\gamma, \theta, p, T}^{\beta}$ to $\mathcal{H}^{\beta+h}\left(L^{2}([0, T]) ; \mathrm{d}\right.$ s) as soon as $0<h<h_{\gamma}$.

$$
\text { Proof. - For } 0<t<t+\tau \leqslant T
$$

$$
J_{t+\tau}^{K_{+}}\left(w_{\beta}\right)(s)-J_{t}^{K_{+}}\left(w_{\beta}\right)(s)=J_{t+\tau}^{K_{+}}\left(1_{(t, t+\tau)}(s) w_{\beta}\right)(s)+J_{t+\tau}^{K_{+}}\left(1_{(0, t)}(s) w_{\beta}\right)(t) .
$$

According to Lemma B.1, the norm of $(u, s) \rightarrow 1_{(t, t+\tau)}(s) w_{\beta}(u, s)$ in $L_{w_{-\gamma q}}^{q}$ is dominated by $C \tau^{\beta+h_{\gamma}}$. Therefore, Proposition 2.9

$$
\left\|J_{t+\tau}^{K_{+}}\left(1_{(t, t+\tau)}(s) w_{\beta}\right)(s)\right\|_{L^{2}([0, T], \mathrm{d} s)} \leqslant C \tau^{\beta+h_{\gamma}}\|K\|_{\gamma, \theta, p, T} .
$$

Since $K_{+} \in A_{\gamma, \theta, p, T}^{\beta}$, then for $h<h_{\gamma}$,

$$
\left\|J_{t+\tau}^{K_{+}}\left(1_{(0, t)}(s) w_{\beta}\right)(t)\right\|_{L^{2}([0, T], \mathrm{d} s)} \leqslant C \tau^{\beta+h}\|K\|_{\beta} .
$$

Combining these two upper bounds yields the desired result.

The following technical results now involve pairs of rough kernels.

LEMMA 2.13. - The mapping $\left(K_{1}, K_{2}\right) \rightarrow\left\langle K_{1}(t, \cdot), K_{2}(t, \cdot)\right\rangle_{L^{2}([0, T])}$ is bilinear continuous from $E_{\gamma, \theta, p, T}^{2}$ to the set of absolutely continuous functions, vanishing at the origin, endowed with the norm

$$
\|f\|=\int_{0}^{T}|\dot{f}|(s) \mathrm{d} s
$$

where $\dot{f}$ is equal almost everywhere to the derivative of $f$.

Proof. - Assume that the kernels can be written, as in the proof of Proposition 2.5,

$$
K_{i}(t, s)=K_{i}(T, s)-\int_{t}^{T} h_{i}(u-s, s) w_{-\gamma}(u+\varepsilon, s) \mathrm{d} u \quad \text { for } s \leqslant t, i=1,2
$$

where $h_{i}(v, s)=\sum_{l=1}^{n} \phi_{l, i}(v) \psi_{l, i}(s)$ and the $\phi_{l, i}, \psi_{l, i} \in L^{\infty}(0, T)$ have disjoint supports. Then the mapping

$$
\Lambda: t \rightarrow\left\langle K_{1}(t, \cdot), K_{2}(t, \cdot)\right\rangle_{L^{2}([0, T])}
$$


is differentiable with derivative

$$
\dot{\Lambda}(t)=K_{1}(t, t) K_{2}(t, t)+\int_{0}^{t}\left(\partial_{1} K_{1}(t, s) K_{2}(t, s)+K_{1}(t, s) \partial_{1} K_{2}(t, s)\right) \mathrm{d} s .
$$

From the domination relation (2.13) we get

$$
|\dot{\Lambda}(t)| \leqslant \frac{\mathrm{d}}{\mathrm{d} t}\left\langle K_{1,+}(t, \cdot), K_{2,+}(t, \cdot)\right\rangle_{L^{2}([0, T])} \cdot
$$

Integrating with respect to $t$ yields, taking into account Proposition 2.10,

$$
\int_{0}^{T}|\dot{\Lambda}(t)| \mathrm{d} t \leqslant\left\langle K_{1,+}(t, \cdot), K_{2,+}(t, \cdot)\right\rangle_{L^{2}([0, T])} \leqslant C\left\|K_{1}\right\|_{\gamma, \theta, p, T}\left\|K_{2}\right\|_{\gamma, \theta, p, T} .
$$

By Proposition 2.5, the space of kernels that can be written as in (2.15) is dense in $E_{\gamma, \theta, p, T}$. This concludes our proof since the space of absolutely continuous functions is closed.

We now associate to a pair $\left(K, K^{\prime}\right)$ of rough kernels an integral operator

$$
J_{2, t}^{K, K^{\prime}}(a)\left(s, s_{1}\right)=a\left(s, s_{1}\right)\left(\int_{s}^{t} K(u, s) \partial_{1} K^{\prime}\left(u, s_{\left.1_{0}\right)} \mathrm{d} u\right) 1_{\left(s_{1}<s\right)}\right.
$$

defined for measurable $a:[0, T]^{2} \rightarrow X$.

LEMMA 2.14. - If $0<h<h_{\gamma}$, then the mapping $J_{2, t}$ is bilinear continuous from $\tilde{\mathcal{H}}^{\beta}(X) \times E_{\gamma, \theta, p, T} \times A_{\gamma, \theta, p, T}^{\beta+h_{\gamma}}$ into $\mathcal{H}^{\beta+2 h}\left(L^{2}\left([0, T]^{2} ; X ; \mathrm{d} s \mathrm{~d} s_{1}\right)\right)$.

Proof. - According to equality (2.14) applied to

$$
\tilde{a}\left(u, s_{1}\right)=K(u, s) a\left(s, s_{1}\right) 1_{\left(s_{1}<s\right)}
$$

we get

$$
1_{\left(s_{1}<s\right)} \Delta_{t, t+\tau}^{0} J_{2}^{K, K^{\prime}}(a)\left(s, s_{1}\right)=J_{t+\tau}^{K^{\prime}}(\tilde{a})(\max (s, t)) .
$$

Using again the dominating kernels, $a \in \tilde{\mathcal{H}}^{\beta}(X)$ and that

$$
\left(s-s_{1}\right)^{\beta} \leqslant\left(u-s_{1}\right)^{\beta} \quad \text { for } 0<s_{1}<s<u
$$

we obtain

$$
\left|\Delta_{t, t+\tau}^{0} J_{2}^{K, K^{\prime}}(a)\left(s, s_{1}\right)\right| \leqslant\|a\|_{\tilde{\mathcal{H}}^{\beta}} J_{t+\tau}^{K_{+}^{\prime}}\left(K_{+}(u, s) w_{\beta}\left(u, s_{1}\right)\right)\left(s_{1}\right) 1_{\left(s>s_{1}\right)} .
$$

According to Proposition 2.10 there exists a constant $C$ such that:

$$
\left\|(u, s) \rightarrow K_{+}(u, s) w_{\beta}\left(u, s_{1}\right)\right\|_{\tilde{\mathcal{H}}^{\beta+h_{\gamma}\left(L^{2}([0, T])\right)}} \leqslant C\|K\|_{\gamma, \theta, p, T} .
$$


Therefore, thanks to Proposition 2.11 we have the upper bound

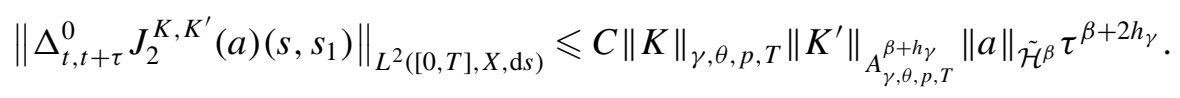

Lemma 2.15. - The mapping $\left(K, K^{\prime}\right) \rightarrow\left\langle K(t+\tau, \cdot)-K(t, \cdot), K^{\prime}(t, \cdot)\right\rangle_{L^{2}([0, T])}$ is bilinear continuous from $A_{\gamma, \theta, p, T}^{\beta} \times E_{\gamma, \theta, p, T}^{2}$ to $\mathbb{R}$ with norm bounded by $C \tau^{\beta+h}$ for any $h \in\left(0, h_{\gamma}\right)$ (we extend $K$ so that $K(u, s)=0$ if $u<s$ ).

Proof. - Thanks to (2.13) we can restrict ourselves to $K_{+}, K_{+}^{\prime}$. we have:

$$
\begin{aligned}
&\left|\left\langle K_{+}(t+\tau, \cdot)-K_{+}(t, \cdot), K_{+}^{\prime}(t, \cdot)\right\rangle_{L^{2}([0, T])}\right| \\
& \quad=\int_{0}^{t} \mathrm{~d} s K_{+}^{\prime}(t, s) \int_{t}^{t+\tau} \mathrm{d} u \partial_{1} K_{+}(u, s) \\
& \quad=\int_{0}^{t} \mathrm{~d} s K_{+}^{\prime}(t, s) \int_{t}^{t+\tau} \mathrm{d} u(u-s)^{-\beta}(u-s)^{\beta} \partial_{1} K_{+}(u, s) \\
& \leqslant \int_{0}^{t} \mathrm{~d} s K_{+}^{\prime}(t, s)(t-s)^{-\beta} \int_{t}^{t+\tau} \mathrm{d} u(u-s)^{\beta} \partial_{1} K_{+}(u, s) \\
&=\int_{0}^{t} \mathrm{~d} s K_{+}^{\prime}(t, s)(t-s)^{-\beta} \Delta_{t, t+\tau}^{0} J^{K K_{+}}\left(w_{\beta}\right)(s) \\
& \leqslant\left(\int_{0}^{t} \mathrm{~d} s K_{+}^{\prime}(t, s)^{2}(t-s)^{-2 \beta}\right)^{1 / 2}\left(\int_{0}^{t} \mathrm{~d} s\left(\Delta_{t, t+\tau}^{0} J^{K_{+}}\left(w_{\beta}\right)(s)\right)^{2}\right)^{1 / 2} \\
& \leqslant\left(C_{1}\left\|K^{\prime}\right\|_{A_{\gamma, \theta, p, T}^{\beta}}\right) \times\left(C_{2} \tau^{\beta+h}\|K\|_{\gamma, \theta, p, T}\right) .
\end{aligned}
$$

The second inequality is Cauchy-Schwarz's inequality, and the last one is due to Hölder's inequality $\left(\theta / 2, \theta^{*} / 2\right)$ for the first factor (since $\beta \theta^{*}<1$ ) and Lemma 2.12 for the second factor. We can now conclude with the help of Remark 2.3.

\section{The space of integrands}

\subsection{A review of basic notions of Malliavin Calculus}

A nice introduction to Malliavin Calculus can be found in Nualart's book [22], but for the sake of completeness, we state here the few definitions and properties we use in this paper.

Let $\Omega$ denote the space $C(I, \mathbb{R}), I=[0, T]$, equipped with the topology of uniform convergence on the compact sets, $\mathcal{F}$ the Borel $\sigma$-field on $\Omega, \mathbb{P}$ the standard Wiener measure, and let $\left\{B_{t}(\omega)=\omega(t), 0 \leqslant t \leqslant T\right\}$. For any $t \geqslant 0$, we define $\mathcal{F}_{t}=\sigma(\omega(s), s \leqslant$ $t) \vee \mathcal{N}$, where $\mathcal{N}$ denotes the class of the elements in $\mathcal{F}$ which have zero $\mathbb{P}$ measure. 
For $h \in L^{2}(I, \mathbb{R})$, we denote by $B(h)$ the Wiener integral

$$
B(h)=\int_{I}\left(h(t), \mathrm{d} B_{t}\right) .
$$

Let $X$ be a separable Hilbert space with norm $|\cdot|_{X}$. (Usually $X=L^{2}\left([0, T]^{i} ; \mathbb{R}\right)$.) Let $\mathcal{S}$ denote the dense subset of $L^{2}(\Omega, \mathcal{F}, \mathbb{P})$ consisting of those classes of random variables of the form:

$$
F=f\left(B\left(h_{1}\right), \ldots, B\left(h_{n}\right)\right),
$$

where $n \in \mathbb{N}, f \in C_{b}^{\infty}\left(\mathbb{R}^{n} ; X\right), h_{1}, \ldots, h_{n} \in L^{2}(I, \mathbb{R})$. If $F$ has the form (3.1), we define its derivative as the process $D F \stackrel{\text { def }}{=}\left\{D_{t} F, t \in I\right\}$ given by

$$
D_{t} F=\sum_{k=1}^{n} \frac{\partial f}{\partial x_{k}}\left(B\left(h_{1}\right), \ldots, B\left(h_{n}\right)\right) h_{k}(t) .
$$

We shall denote by $\mathbf{D}^{1, \alpha}$ the closure of $\mathcal{S}$ with respect to the norm

$$
\|F\|_{1, \alpha}=\|F\|_{\alpha}+\|\| D F\left\|_{L^{2}(I)}\right\|_{\alpha} .=\mathbb{E}\left[|F|^{\alpha}\right]^{1 / \alpha}+\mathbb{E}\left[\left(\int_{0}^{t}\left|D_{u} F\right|^{2} \mathrm{~d} u\right)^{\alpha / 2}\right]^{1 / \alpha} .
$$

The higher order derivative $D^{n} F$ are defined inductively, and the space $\mathbf{D}^{n, \alpha}$ is the closure of $\mathcal{S}$ under the norm

$$
\|F\|_{n, \alpha}=\|F\|_{\alpha}+\left\|\sum_{i=1}^{n}\right\| D^{i} F\left\|_{L^{2}\left(I^{i}\right)}\right\|_{\alpha} .
$$

Then, define $\delta$, the Skorokhod integral with respect to $W$, as the adjoint of $D$, i.e., $\operatorname{Dom}(\delta)$ is the set of $u \in L^{2}(\Omega \times I)$ such that there exists a constant $c$ with

$$
\left|\mathbb{E}\left[\int_{I} D_{t} F u_{t} \mathrm{~d} t\right]\right| \leqslant c\|F\|_{2}, \quad \forall F \in \mathcal{S} .
$$

If $u \in \operatorname{Dom} \delta, \delta(u)$ is defined as the unique element of $L^{2}(\Omega)$ which satisfies

$$
\mathbb{E}[\delta(u) F]=\mathbb{E}\left[\int_{I} D_{t} F u_{t} \mathrm{~d} t\right], \quad \forall F \in \mathcal{S} .
$$

In order to prevent a confusion between $\delta$, an admissible parameter, and $\delta$ the Skorokhod integral, we shall from now on use the same notations for the Ito and the Skorokhod integral, that is:

$$
\delta(u)=\int_{0}^{T} u(s) \mathrm{d} B_{s}
$$


Let $\mathbf{L}^{1,2}$ be the Hilbert space $L^{2}\left(I ; \mathbf{D}^{1,2}\right)$ endowed with the norm

$$
\|u\|_{\mathbf{L}^{1,2}}^{2}=\mathbb{E}\left[\int_{I} u_{t}^{2} \mathrm{~d} t+\int_{I \times I}\left(D_{v} u_{t}\right)^{2} \mathrm{~d} v \mathrm{~d} t\right] .
$$

We have $\mathbf{L}^{1,2} \subset \operatorname{Dom} \delta$, and for $u \in \mathbf{L}^{1,2}$

$$
\mathbb{E}\left[\left(\int_{0}^{T} u(s) \mathrm{d} B_{s}\right)^{2}\right]=\mathbb{E}\left[\int_{I} u_{t}^{2} \mathrm{~d} t\right]+\mathbb{E}\left[\int_{I} \int_{I} D_{t} u_{s} D_{s} u_{t} \mathrm{~d} s \mathrm{~d} t\right] \leqslant\|u\|_{\mathbf{L}^{1,2}}^{2}
$$

Note that $\left\{u \in L^{2}(\Omega \times I) ; u\right.$ is $\mathcal{F}_{t}$ progressively measurable $\} \subset \operatorname{Dom} \delta$, and for such a $u$, $\delta(u)$ coincide with the usual Itô integral. Note that when $u$ is progressively measurable, $D_{s} u_{t}=0$ for $s>t$, so (3.2) is consistent with the formula in the adapted case.

Let $\mathbf{L}^{2,2}$ be the Hilbert space $L^{2}\left(I^{2}, \mathbf{D}^{2,2}\right)$ endowed with the norm

$$
\|u\|_{\mathbf{L}^{2,2}}^{2}=\underset{I^{2}}{\mathbb{E}}\left[\int_{I^{3}} u^{2}(t, s) \mathrm{d} t \mathrm{~d} s+\int_{I^{4}}\left(D_{v} u(t, s)\right)^{2} \mathrm{~d} v \mathrm{~d} t \mathrm{~d} s+\int_{v, r}\left(D^{2} u(t, s)\right)^{2} \mathrm{~d} t \mathrm{~d} s \mathrm{~d} v \mathrm{~d} r\right] .
$$

We have $\mathbf{L}^{2,2} \subset \operatorname{Dom}(\delta \circ \delta)$ and for $u \in \mathbf{L}^{2,2}$

$$
\mathbb{E}\left[\int_{0}^{T}\left(\int_{0}^{s} u\left(s, s_{1}\right) \mathrm{d} B_{s_{1}}\right) \mathrm{d} B_{s}\right] \leqslant 2\|u\|_{\mathbf{L}^{2,2}}^{2}
$$

Property $P$ (Integration by parts formula). - Suppose that $u$ belongs to $\mathbf{L}^{1,2}$. Let $F$ be a random variable belonging to $\mathbf{D}^{1,2}$ such that $\mathbb{E}\left[F^{2} \int_{I} u_{t}^{2} \mathrm{~d} t\right]<\infty$, then

$$
\int_{I} F u_{t} \mathrm{~d} B_{t}=F \int_{I} u_{t} \mathrm{~d} B_{t}-\int_{I} D_{t} F u_{t} \mathrm{~d} t
$$

in the sense that $F u$ belong to $\operatorname{Dom}(\delta)$ if and only if the right-hand side of (3.5) belongs to $L^{2}(\Omega)$.

For $m=1,2$, let $\mathcal{C}^{\beta} \mathbf{L}^{m, 2}(0, t)=\mathcal{H}^{\beta}\left(\mathbf{D}^{m, 2}\right) \cap \mathbf{L}^{m, 2}$ be the space of processes $a \in$ $\mathbf{L}^{m, 2}(0, t)$ such that for a constant $C$

$$
\|a(u)-a(v)\|_{\mathbf{D}^{m, 2}} \leqslant C|u-v|^{\beta} \quad(0 \leqslant u, v \leqslant t) .
$$

\subsection{The space of good integrands}

Recall that $(p, \gamma, \theta)$ is a set of admissible parameters, that is

$$
\begin{aligned}
& 1<p<2, \quad \frac{1}{p}+\frac{1}{q}=1, \quad \frac{2}{q}>\gamma>\frac{1}{q}, \quad \delta=\frac{2 p}{2-p} \\
& h_{\gamma}=\frac{1}{\theta^{*}}<\frac{2}{q}-\gamma, \quad \frac{1}{\theta / 2}+\frac{1}{\theta^{*} / 2}=1 .
\end{aligned}
$$


The space $G I_{\gamma, \theta, q, t}$ is the space of adapted processes $a \in \mathbf{L}^{1,2}(0, t)$ such that $\|a\|_{\gamma, \theta, q, t}<$ $\infty$ where

$$
\begin{aligned}
\|a\|_{\gamma, \theta, q, t} \stackrel{\text { def }}{=} & \left(\int_{0<s<u<t}\left(\|a(u)-a(s)\|_{\mathbf{D}^{1,2}}(u-s)^{-\gamma}\right)^{q} \mathrm{~d} u \mathrm{~d} s\right)^{1 / q} \\
& +\left(\int_{0}^{t}\|a(s)\|_{2}^{\theta^{*}} \mathrm{~d} s\right)^{1 / \theta^{*}}
\end{aligned}
$$

(we hope that the use of the same notations form norms of kernels and norms of integrands will not confuse the reader). With the notations introduced in the previous section,

$$
\|a\|_{\gamma, \theta, q, t}=\left\|\Delta^{0} a\right\|_{L_{w_{-\gamma q}}^{q}\left(\mathbf{D}^{1,2)}\right.}+\|a\|_{L^{\theta^{*}\left(L^{2}(\Omega)\right)}} .
$$

Observe that if $a \in \mathbf{C}^{\beta} \mathbf{L}^{1,2}(0, t)$ is adapted and bounded, and if $\beta>\gamma-\frac{1}{q}$, then $a \in G I_{\gamma, \theta, q, t}$.

Finally, we need to introduce the subset $C G I_{\gamma, \theta, q, t}$ of processes $a \in G I_{\gamma, \theta, q, t}$ such that

$$
\int_{0}^{t} \int_{s}^{t} \mathbb{E}\left[\left(D_{s} a(u)\right)^{2}\right]^{q / 2}(u-s)^{-\gamma q} \mathrm{~d} u \mathrm{~d} s<+\infty
$$

which we endow with the norm

$$
|\|a\||_{\gamma, \theta, q, t}=\|a\|_{\gamma, \theta, q, t}+\|D a\|_{L_{w-\gamma q}^{q}\left(L^{2}(\Omega)\right)} .
$$

\section{Integration of deterministic functions}

We recall that to a rough kernel $K$ we associate the integral operator defined for a measurable $a$ on $(0, t)$

$$
I_{t} a(s)=K(t, s) a(s)+\int_{s}^{t}(a(u)-a(s)) \partial_{1} K(u, s) \mathrm{d} u \quad(0<s<t)
$$

as soon as the integral on the right-hand side makes sense for almost every $s$ in $(0, t)$.

When $K$ is a smooth kernel, we have

$$
I_{t} a(s)=K(s, s) a(s)+\int_{s}^{t} a(u) \partial_{1} K(u, s) \mathrm{d} u \quad(0<s<t) .
$$

We shall also consider the integral operator

$$
J_{t} a(s)=\int_{s}^{t} a(u, s) \partial_{1} K(u, s) \mathrm{d} u \quad(0<s<t) .
$$


We end this section by the definition of the integral of a deterministic function. Since $K(t, s)=I_{t} 1_{[0, t]}(s)$, we have, for $s<t$,

$$
\begin{aligned}
\operatorname{Cov}\left(W_{K}(s), W_{K}(t)\right) & =\mathbb{E}\left[\int_{0}^{t} K(t, u) \mathrm{d} B_{u} \int_{0}^{s} K(s, v) \mathrm{d} B_{v}\right] \\
& =\left\langle I_{t} 1_{[0, t]}, I_{t} 1_{[0, s]}\right\rangle_{L^{2}},
\end{aligned}
$$

where $\langle,\rangle_{L^{2}}$ denotes the inner product of $L^{2}\left(\mathbb{R}_{+}\right)$.

Accordingly, we define $L_{K}^{2}(0, t)=I_{t}^{-1}\left(L^{2}(0, t)\right)$ and endow it with the inner product

$$
\langle f, g\rangle_{L_{K}^{2}(0, t)} \stackrel{\text { def }}{=}\left\langle I_{t} f, I_{t} g\right\rangle_{L^{2}} .
$$

$\overline{L_{K}^{2}(0, t)}$ is the closure of $L_{K}^{2}(0, t)$ with respect to $\langle\cdot, \cdot\rangle_{L_{K}^{2}(0, t)}$. Given $f \in L_{K}^{2}(0, t)$, it is natural to define

$$
\int_{0}^{t} f(s) \mathrm{d} W_{K}(s)=\int_{0}^{t} I_{t} f(u) \mathrm{d} B_{u} .
$$

This is clearly an isometry between $\overline{L_{K}^{2}(0, t)}$ and the Gaussian space generated by $\left(W_{K}(s), 0 \leqslant s \leqslant t\right)$. It is interesting to note that for $f \equiv 1$ we obtain

$$
\int_{0}^{t} \mathrm{~d} W_{K}(s)=W_{K}(t)
$$

\section{Regularity of sample paths}

Proposition 5.1. - Assume that $K \in E_{\gamma, \theta, p, t}$. Then, almost surely, the sample paths of $W_{K}$ are Hölder continuous of index $\tau \in\left(0, h_{\gamma}\right)$. More precisely, if $\alpha>\frac{1}{h_{\gamma}}$ and $\tau \in\left[0, h_{\gamma}-\frac{1}{\alpha}[\right.$ then for some constant $C$

$$
\left\|\sup _{0 \leqslant u<v \leqslant t} \frac{\left|W_{K}(v)-W_{K}(u)\right|}{|v-u|^{\tau}}\right\|_{\alpha} \leqslant C\|K\|_{\gamma, \theta, p, t} .
$$

Proof. - Since $W_{K}$ is a Gaussian process with covariance

$$
R_{K}(u, v)=\int_{0}^{\min (u, v)} K(v, s) K(u, s) \mathrm{d} s .
$$

Proposition 2.10 yields for $0 \leqslant u<v<t$

$$
\left\|W_{K}(v)-W_{K}(u)\right\|_{2}=\|K(v, \cdot)-K(u, \cdot)\|_{L^{2}(0, t)} \leqslant C|v-u|^{h_{\gamma}}\|K\|_{\gamma, \theta, p, t} .
$$

Then, we use Kolmogorov's Continuity Lemma in the special case of Gaussian processes, as described by the next lemma. 
Lemma 5.2. - Let $(Z(t), 0 \leqslant t \leqslant T)$ be a centered Gaussian process such that for some $\delta>0$

$$
\mathbb{E}\left[(Z(t)-Z(s))^{2}\right] \leqslant C(T)|t-s|^{\delta} \quad(0 \leqslant s, t \leqslant T) .
$$

Then $Z$ has a modification (denoted by the same letter $Z$ ) with Hölder continuous paths of order $\lambda$ for each $\lambda \in[0, \delta / 2[$.

Furthermore, for every $p \geqslant \sup (1,2 / \delta)$ we have

$$
\begin{aligned}
& \left\|\sup _{0 \leqslant s, t \leqslant T}|Z(t)-Z(s)|\right\|_{p} \leqslant C(T)^{1 / 2} \frac{2^{1+1 / p}}{1-2^{1 / 2 p-\delta / 2}} T^{\delta / 2} m_{p}, \\
& \left\|\sup _{0 \leqslant s<t \leqslant T} \frac{|Z(t)-Z(s)|}{(t-s)^{\lambda}}\right\|_{p} \leqslant C^{\prime}(\lambda, \delta, p, T) C(T)^{1 / 2} \quad(0 \leqslant \lambda<\delta / 2-1 / p) .
\end{aligned}
$$

Where $m_{p}^{p}$ is the pth moment of the absolute value of a standard Gaussian random variable.

Since the proof of this lemma uses the explicit constant of Kolmogorov's Lemma, we feel compelled to state here the version of this lemma that we use, which can be found in [10], Section XXIII, numbers 19 and 20.

LEMmA 5.3. - Let $(X(t), 0 \leqslant t \leqslant T)$ be a real process such that for some $\sigma>0$ and some $p \geqslant 1$

$$
\mathbb{E}\left[\left|X_{t}-X_{s}\right|^{p}\right] \leqslant C|t-s|^{1+\sigma} \quad(0 \leqslant s, t \leqslant T) .
$$

Then for every $\lambda \in[0, \sigma / p[$ the random variable

$$
M_{\lambda}=\sup \left\{\frac{\left|X_{t}-X_{s}\right|}{|t-s|^{\lambda}}: s, t \in \mathbb{Q}, s \neq t, 0 \leqslant s, t \leqslant T\right\}
$$

is almost surely finite. More precisely,

$$
\left\|M_{\lambda}\right\|_{p} \leqslant C^{1 / p} \frac{2^{\lambda+1+1 / p}}{1-2^{\lambda-\sigma / p}} T^{(1+\sigma-\lambda) / p} .
$$

Proof of Lemma 5.2. - For every $p \geqslant 1$, since $Z(t)-Z(s)$ is a centered Gaussian random variable, we have

$$
\mathbb{E}\left[|Z(t)-Z(s)|^{p}\right] \leqslant m_{p}^{p} C(T)^{p / 2}|t-s|^{\delta p / 2} \quad(0 \leqslant s, t, T) .
$$

If $p>\sup (1,2 / \delta)$, then we obtain the upper bound on the $p$ th moment, and the fact that $Z$ has Hölder continuous paths for every $\lambda \in[0, \delta / 2-1 / p[$.

COROLlary 5.4. - Assume that for some $p \geqslant 1, K \in E_{\gamma, \theta, p, t}$. Then there exists a sequence of kernels $\left(K_{n}, n \in \mathbb{N}\right)$ in $F_{\gamma, \theta, p, t}$ such that

$$
\sup _{s \leqslant t}\left|W_{K}(s)-W_{K_{n}}(s)\right| \rightarrow 0 \quad \text { in } L^{2} \text { and a.s. }
$$


Proof. - There exists a sequence of kernels $\left(K_{n}, n \in \mathbb{N}\right)$ in $F_{\gamma, \theta, p, t}$ such that $\left\|K_{n}-K\right\|_{\gamma, \theta, p, t} \rightarrow 0$. We let

$$
M_{n}=\sup _{s \leqslant t}\left|W_{K}(s)-W_{K_{n}}(s)\right| .
$$

Proposition 5.1 yields, for $\alpha>\frac{1}{h_{\gamma}}$ and $\tau=0$,

$$
\left\|M_{n}\right\|_{\alpha} \leqslant C\left\|K-K_{n}\right\|_{\gamma, \theta, p, t} \rightarrow 0 .
$$

By taking a subsequence, we can ensure that $\sum\left\|M_{n}\right\|_{\alpha}^{\alpha}<+\infty$, and thus obtain, by a Borel-Cantelli argument, the almost sure convergence of $M_{n}$ to 0 .

\section{Construction of the stochastic integral}

The first step of our construction is to identify the semi-martingale integral $\int a(s) \times$ $\mathrm{d} W_{K}(s)$, for $a$ adapted and $W_{K}$ a semi-martingale, with a sum of (Skorokhod) integrals of $a$ and its stochastic gradient with respect to the driving Brownian motion $B$ and time.

Proposition 6.1. - Assume that $a \in G I_{\gamma, \theta, q, t}$ and $K$ is a semi-martingale kernel in $E_{\gamma, \theta, p, t}$. Then

(i) The process $I_{t}(a)(\cdot)$ is in $\mathbf{L}^{1,2}(0, t)$ and

$$
\left\|\int_{0}^{t} I_{t}(a)(s) \mathrm{d} B_{s}\right\| \leqslant\left\|I_{t}(a)\right\|_{\mathbf{L}^{1,2}(0, t)} \leqslant\|a\|_{\gamma, \theta, q, t}\|K\|_{\gamma, \theta, p, t} .
$$

(ii) We have the decomposition

$$
\int_{0}^{t} a(s) \mathrm{d} W_{K}(s)=\int_{0}^{t} I_{t} a(s) \mathrm{d} B_{s}+\int_{0}^{t}\left(\int_{s}^{t} D_{s} a(u) \partial_{1} K(u, s) \mathrm{d} u\right) \mathrm{d} s .
$$

(iii) If furthermore $a \in C G I_{\gamma, \theta, q, t}$ then the process

$$
s \rightarrow J_{t}(D .(a)(\cdot))(s)=\int_{s}^{t} D_{s} a(u) \partial_{1} K(u, s) \mathrm{d} u
$$

satisfies

$$
\begin{aligned}
\left\|\int_{0}^{t} J_{t}(D .(a)(\cdot))(s)\right\|_{L^{2}(\Omega)} & \leqslant \int_{0}^{t}\left\|J_{t}(D .(a)(\cdot))(s)\right\|_{L^{2}(\Omega)} \mathrm{d} s \\
& \leqslant C|\|a\||_{\gamma, \theta, q, t}\|K\|_{\gamma, \theta, p, t}
\end{aligned}
$$


and we have the upper bound

$$
\left\|\int_{0}^{t} a(s) \mathrm{d} W_{K}(s)\right\|_{L^{2}(\Omega)} \leqslant C|\|a\||_{\gamma, \theta, q, t}\|K\|_{\gamma, \theta, p, t} .
$$

Proof. - (i) On the one hand, Lemma 6.2(ii) implies that $J_{t}(a)(\cdot) \in \mathbf{L}^{1,2}(0, t)$ and

$$
\left\|J_{t}(a)\right\|_{\mathbf{L}^{1,2}(0, t)} \leqslant\|a\|_{L_{w_{-\gamma q}}^{q}\left(\mathbf{D}^{1,2}\right)}\|K\|_{\gamma, \theta, p, t} .
$$

On the other hand, the second part of Proposition 2.9 implies that

$$
\|K(t, \cdot) a(\cdot)\|_{L^{2}\left([0, T], L^{2}(\Omega, \mathbb{P})\right)} \leqslant C\|a\|_{L^{\theta^{*}\left(L^{2}(\Omega)\right)}}\|K\|_{\gamma, \theta, p, T} .
$$

We conclude by combining these two results.

(ii) Recall from Proposition 2.5 that $W_{K}$ is a semi-martingale with decomposition

$$
\mathrm{d} W_{K}(s)=K(s, s) \mathrm{d} B_{s}+W_{\partial_{1} K}(s) \mathrm{d} s .
$$

Therefore, since $a$ is adapted and in $\mathbf{L}^{1,2}(0, t)$, we have that

$$
\begin{aligned}
\int_{0}^{t} a(s) \mathrm{d} W_{K}(s) & =\int_{0}^{t} a(s) K(s, s) \mathrm{d} B_{s}+\int_{0}^{t} a(s) W_{\partial_{1} K}(s) \mathrm{d} s \\
& =\int_{0}^{t} a(s) K(s, s) \mathrm{d} B_{s}+\int_{0}^{t} a(u)\left(\int_{0}^{u} \partial_{1} K(u, s) \mathrm{d} B_{s}\right) \mathrm{d} u .
\end{aligned}
$$

We now apply the integration by parts formula of Malliavin Calculus

$$
\begin{aligned}
\int_{0}^{t} a(s) \mathrm{d} W_{K}(s)= & \int_{0}^{t} a(s) K(s, s) \mathrm{d} B_{s}+\int_{0}^{t}\left(\int_{0}^{u} a(u) \partial_{1} K(u, s) \mathrm{d} B_{s}\right) \mathrm{d} u \\
& +\int_{0}^{t}\left(\int_{0}^{u} D_{s} a(u) \partial_{1} K(u, s) \mathrm{d} s\right) \mathrm{d} u .
\end{aligned}
$$

Since $M^{2} \stackrel{\text { def }}{=} \sup _{x \leqslant t} \int_{0}^{x} \partial_{1} K(x, u)^{2} \mathrm{~d} u<+\infty$, we can apply Fubini's Theorem to the third term on the right-hand side of (6.3) to obtain

$$
\int_{0}^{t}\left(\int_{0}^{u} D_{s} a(u) \partial_{1} K(u, s) \mathrm{d} s\right) \mathrm{d} u=\int_{0}^{t}\left(\int_{s}^{t} D_{s} a(u) \partial_{1} K(u, s) \mathrm{d} u\right) \mathrm{d} s .
$$

Indeed,

$$
\mathbb{E}\left[\int_{0}^{t}\left(\int_{0}^{u}\left|D_{s} a(u)\right|\left|\partial_{1} K(u, s)\right| \mathrm{d} s\right) \mathrm{d} u\right]
$$




$$
\begin{aligned}
& \leqslant M \mathbb{E}\left[\int_{0}^{t}\left(\int_{0}^{u}\left|D_{s} a(u)\right|^{2} \mathrm{~d} s\right)^{1 / 2} \mathrm{~d} u\right] \\
& \leqslant M\|a\|_{\mathbf{L}^{1,2}} .
\end{aligned}
$$

The anticipating Fubini's Stochastic Theorem (see Theorem 3.1 of Leon [17]) yields that

$$
\int_{0}^{t}\left(\int_{0}^{s} a(u) \partial_{1} K(u, s) \mathrm{d} B_{s}\right) \mathrm{d} u=\int_{0}^{t}\left(\int_{s}^{t} a(u) \partial_{1} K(u, s) \mathrm{d} u\right) \mathrm{d} B_{s} .
$$

Indeed, all we need to do is to consider the finite measure $\mu(\mathrm{d} x)=\mathrm{d} x 1_{[0, t]}(x)$ on $X=[0, t]$ and the measurable function

$$
\phi(x, u, \omega)=a(x, \omega) \partial_{1} K(x, u) \quad(x, u \leqslant t) .
$$

It is clear that for fixed $x \in[0, t], \phi(x): u \rightarrow \phi(x, u, \cdot)$ is in $\mathbf{L}^{1,2}(0, t)$, with $D_{v} \phi(x, u)=$ $D_{v} a(x) \partial_{1} K(x, u)$ and

$$
\begin{aligned}
\|\phi(x)\|_{\mathbf{L}^{1,2}}^{2} & =\mathbb{E}\left[\int_{0}^{t}\left(\phi(x, u)^{2}+\int_{0}^{t}\left(D_{v} \phi(x, u)\right)^{2} \mathrm{~d} v\right) \mathrm{d} u\right] \\
& =\int_{0}^{x} \mathrm{~d} u \partial_{1} K(x, u)^{2} \mathbb{E}\left[a(x)^{2}+\int_{0}^{x}\left(D_{v} a(x)\right)^{2} \mathrm{~d} v\right] .
\end{aligned}
$$

Therefore the Bochner integral $\int_{X} \phi(x) \mathrm{d} \mu(x)$ is well defined since

$$
\begin{aligned}
& \left(\int \mu(\mathrm{d} x)\|\phi(x)\|_{\mathbf{L}^{1,2}}\right)^{2} \\
& \quad \leqslant \mu(X) \int \mu(\mathrm{d} x)\|\phi(x)\|_{\mathbf{L}^{1,2}}^{2} \\
& \quad \leqslant \mu(X) \mathbb{E}\left[\int_{0}^{t} \mathrm{~d} x\left(a(x)^{2}+\int_{0}^{x}\left(D_{v} a(x)\right)^{2} \mathrm{~d} v\right) \int_{0}^{x} \partial_{1} K(x, u)^{2} \mathrm{~d} u\right] \\
& \quad \leqslant \mu(X)\left(\sup _{x \leqslant t} \int_{0}^{x} \partial_{1} K(x, u)^{2} \mathrm{~d} u\right)\|a\|_{\mathbf{L}^{1,2}(0, t)}<+\infty .
\end{aligned}
$$

(iii) The first part is a consequence of the first part of Proposition 2.9 applied to the process $\tilde{a}(u, s)=D_{s} a(u)$. We conclude by combining this upper bound with (i).

LEMMA 6.2.-

(i) For every $a \in \mathbf{L}^{1,2}(0, t)$ such that $s \rightarrow \int_{s}^{t} a(v) \partial_{1} K(v, s) \mathrm{d} v$ is in $\mathbf{L}^{1,2}$ we have the commutation relation

$$
D_{u} \int_{s}^{t} a(v) \partial_{1} K(v, s) \mathrm{d} v=\int_{s}^{t} D_{u} a(v) \partial_{1} K(v, s) \mathrm{d} v .
$$


(ii) The application $(a, K) \rightarrow J_{t}^{K}(a)$ is bilinear continuous from $\left(\mathbf{L}^{1,2}(0, t) \cap\right.$ $\left.L_{w_{-\gamma q}}^{q}\left(\mathbf{D}^{m, 2}\right)\right) \times E_{\gamma, \theta, p, T}$ to $\mathbf{L}^{m, 2}$ with $m=0,1,2$.

Proof. - (i) The simplest way to see this property is to use the Wiener chaos expansion of $a$

$$
a(s)=\sum_{m \geqslant 0} \Lambda_{m}\left(f_{m}(\cdot, s)\right),
$$

where $\Lambda_{m}$ denotes the multiple Wiener integral and $f_{m}\left(s_{1}, \ldots, s_{m}, s\right)$ are square integrable kernels symmetric in the first $m$ variables. By linearity, we can restrict ourselves to the case $a=\Lambda_{m}\left(f_{m}(\cdot, s)\right)$ for some $m \geqslant 1$.

On the one hand, Fubini's Stochastic Theorem implies that

$$
\begin{aligned}
\int_{s}^{t} a(v) \partial_{1} K(v, s) \mathrm{d} v & =\int_{s}^{t} \mathrm{~d} v \partial_{1} K(v, s) \Lambda_{m}\left(f_{m}(\bullet, v)\right) \\
& =\Lambda_{m}\left(\bullet \rightarrow \int_{s}^{t} \partial_{1} K(v, s) f_{m}(\bullet, v) \mathrm{d} v\right) .
\end{aligned}
$$

Therefore,

$$
D_{u} \int_{s}^{t} a(v) \partial_{1} K(v, s) \mathrm{d} v=m \Lambda_{m-1}\left(\bullet \rightarrow \int_{s}^{t} \partial_{1} K(v, s) f_{m}(\bullet, u, v) \mathrm{d} v\right) .
$$

On the other hand,

$$
\begin{aligned}
\int_{s}^{t} D_{u} a(v) \partial_{1} K(v, s) \mathrm{d} v & =\int_{s}^{t} \partial_{1} K(v, s) m \Lambda_{m-1}\left(f_{m}(\bullet, u, v)\right) \mathrm{d} v \\
& =m \Lambda_{m-1}\left(\bullet \rightarrow \int_{s}^{t} \partial_{1} K(v, s) f_{m}(\bullet, u, v) \mathrm{d} v\right) .
\end{aligned}
$$

(ii) For $t \in[0, T]$, Proposition 2.9 implies

$$
\begin{aligned}
\| s & \rightarrow J_{t}^{K}(a(\cdot, s))(s)\left\|_{L^{2}(\Omega \times[0, T])}+\right\|(r, s) \rightarrow J_{t}^{K}\left(D_{r} a(\cdot, s)\right)(s) \|_{L^{2}\left(\Omega \times[0, T]^{2}\right)} \\
& \leqslant C\|a\|_{L_{w-\gamma q}^{q}\left(\mathbf{D}^{1,2}\right)}\|K\|_{\gamma, \theta, p, T} .
\end{aligned}
$$

We shall now use a by product of the proof of Proposition 2.5, namely the existence of a sequence of semi-martingale kernels $\left(\Lambda_{n}\right)_{n \in \mathbb{N}}$ given by

$$
\begin{aligned}
& \Lambda_{n}(r, s)=K(T, s)-\int_{r}^{T} h^{n}(u-s, s)\left(u+\frac{1}{n}-s\right)^{-\gamma} \mathrm{d} u, \\
& h^{n}(v, s)=\sum_{i=0}^{N} \phi_{i}^{n}(v) \psi_{i}^{n}(s),
\end{aligned}
$$


where $\phi_{i}^{n}, \psi_{i}^{n}$ are in $L^{\infty}$, the $i \rightarrow \phi_{i}^{n}$ have disjoint support and $\lim _{n \rightarrow \infty}\left\|K-\Lambda_{n}\right\|_{\gamma, \theta, p, T}$ $=0$.

It is clear (see (i)) that for every $n$, the random variable

$$
J_{t}^{\Lambda_{n}}(a(\cdot, s))(s)=\int_{s}^{t} a(u, s) h^{n}(u-s, s)\left(u+\frac{1}{n}-s\right)^{-\gamma} \mathrm{d} u
$$

belongs to $\mathbf{D}^{1,2}$ and that

$$
D_{r} J_{t}^{\Lambda_{n}}(a(\cdot, s))(s)=J_{t}^{\Lambda_{n}}\left(D_{r} a(\cdot, s)\right)(s) .
$$

From the inequality (6.4) applied to the kernels $\Lambda_{n}-K, \Lambda_{n}-\Lambda_{m}$ we deduce that the sequence $\left(J_{t}^{\Lambda_{n}}(a(\cdot, s))(s), s \in[0, T]\right)$ is a Cauchy sequence in $\mathbf{L}^{1,2}(0, T)$ that converges to $\left(J_{t}^{K}(a(\cdot, s))(s), s \in[0, T]\right)$ in $L^{2}(\Omega \times[0, T])$.

Thanks to (6.5), we obtain also that $\left(D_{r} J_{t}^{\Lambda_{n}}(a(\cdot, s))(s),(s, r) \in[0, T]^{2}\right)$ converges to $\left(J^{K}\left(D_{r} a(\cdot, s)\right)(s),(s, r) \in[0, T]^{2}\right)$ in $L^{2}\left(\Omega \times[0, T]^{2}\right)$.

Therefore, $\left(J_{t}^{K}(a(\cdot, s))(s), s \in[0, T]\right)$ is in $\mathbf{L}^{1,2}(0, T)$ and we have the commutation relation.

$$
D_{r} J_{t}^{K}(a(\cdot, s))(s)=J_{t}^{K}\left(D_{r} a(\cdot, s)\right)(s) .
$$

It is now clear that (6.4) gives the proof of the second part of the lemma for $m=1$. For $m=2$, we only need to replace $a$ by $D a$.

Combining the density of $F_{\gamma, \theta, p, t}$ in $E_{\gamma, \theta, p, t}$ (see Proposition 2.5) with Proposition 6.1 yields that the continuous bilinear operator $\lambda_{t}: C G I_{\gamma, \theta, q, t} \times F_{\gamma, \theta, p, t} \rightarrow L^{2}$ :

$$
\lambda_{t}(a, K)=\int_{0}^{t} a(s) \mathrm{d} W_{K}(s)
$$

can be uniquely extended to an operator $\tilde{\lambda}_{t}: \overline{C G I_{\gamma, \theta, q, t}} \times E_{\gamma, \theta, p, t} \rightarrow L^{2}$, defined on the closure of this product space.

Definition 6.3. - For $a \in \overline{C G I_{\gamma, \theta, q, t}}$ and $K \in E_{\gamma, \theta, p, t}$ the stochastic integral of $a$ with respect to $W_{K}$ is defined to be $\tilde{\lambda}_{t}(a, K)$ and denoted by $\int_{0}^{t} a(s) \mathrm{d} W_{K}(s)$. By construction we have the decomposition (6.1) and the upper bound (6.2).

\section{The stochastic integral as a process}

We shall exhibit assumptions that ensure that the process $t \rightarrow \int_{0}^{t} a(s) \mathrm{d} W_{K}(s)$ has a continuous modification.

THEOREM 7.1. - Assume that for some $\beta>0$ such that $\beta+h_{\gamma}>\frac{1}{2}$ :

- $K \in A_{\gamma, \theta, p, T}^{\beta}$.

- The adapted integrand a is in $\mathbf{C}^{\beta} \mathbf{L}^{1,2}(0, T)$.

- $\sup _{s \leqslant T}|a| \in L^{\alpha}$, for $\alpha h_{\gamma}>1$. 
Then the process $t \rightarrow \int_{0}^{t} I_{t} a(s) \mathrm{d} B_{s}$ has a continuous modification on $[0, T]$.

If, furthermore, $\alpha \in C G I_{\gamma, \theta, q, T}$ then $t \rightarrow \int_{0}^{t} a(s) \mathrm{d} W_{K}(s)$ has a continuous modification on $[0, T]$.

Proof. - Recall that the stochastic integral may be represented as

$$
\begin{aligned}
& \int_{0}^{t} I_{t}(a)(s) \mathrm{d} B_{s}=X(t)+Y(t), \\
& X(t)=\int_{0}^{t} K(t, s) a(s) \mathrm{d} B_{s}, \\
& Y(t)=\int_{0}^{t} J_{t}\left(\Delta^{0} a\right)(s) \mathrm{d} B_{s} .
\end{aligned}
$$

The continuity of $X$ is established via Kolmogorov's continuity criterion. More precisely for $\alpha>0$ big enough, there exists $\eta>0$ and $C=C(T, \alpha)$ such that

$$
\mathbb{E}\left[|X(t+\tau)-X(t)|^{\alpha}\right] \leqslant C \tau^{1+\eta} \quad(0 \leqslant t<t+\tau \leqslant T) .
$$

First we write

$$
\begin{aligned}
X(t+\tau)-X(t)= & \int_{0}^{t}(K(t+\tau, s)-K(t, s)) a(s) \mathrm{d} B_{s} \\
& +\int_{t}^{t+\tau} K(t+\tau, s) a(s) \mathrm{d} B_{s} .
\end{aligned}
$$

Since $a$ is adapted, we can apply Burkholder-Davis-Gundy inequalities to the martingales

$$
\begin{aligned}
& r \rightarrow \int_{0}[K(t+\tau, s)-K(t, s)] 1_{(0, t)}(s) a(s) \mathrm{d} B_{s}, \\
& r \rightarrow \int_{0} K(t+\tau, s) 1_{(t, t+\tau)}(s) a(s) \mathrm{d} B_{s}
\end{aligned}
$$

to obtain, for a constant $C_{\alpha}$, the upper bound

$$
\begin{aligned}
\mathbb{E}\left[|X(t+\tau)-X(t)|^{\alpha}\right]^{1 / \alpha} \leqslant & C_{\alpha} \mathbb{E}\left[\left(\int_{0}^{t}[K(t+\tau, s)-K(t, s)]^{2} a(s)^{2} \mathrm{~d} s\right)^{\alpha / 2}\right]^{1 / \alpha} \\
& +C_{\alpha} \mathbb{E}\left[\left(\int_{t}^{t+\tau} K(t+\tau, s)^{2} a(s)^{2} \mathrm{~d} s\right)^{\alpha / 2}\right]^{1 / \alpha} .
\end{aligned}
$$

The integrability assumptions on $a$, and Proposition 5.1 implies, for $\alpha>2$, 


$$
\begin{aligned}
\|X(t+\tau)-X(t)\|_{L^{\alpha}(\Omega)} & \leqslant C_{\alpha}\left\|\sup _{s \leqslant T}|a(s)|\right\|_{L^{\alpha}(\Omega)}\|K(t+\tau, \cdot)-K(t, \cdot)\|_{L^{2}([0, T])} \\
& \leqslant C\left\|\sup _{s \leqslant T} \mid a(s)\right\|\left\|_{L^{\alpha}(\Omega)}\right\| K \|_{\gamma, \theta, p, T} \tau^{h_{\gamma}} .
\end{aligned}
$$

Therefore we only need to assume also $\alpha h_{\gamma}>1$ to obtain (7.1). The existence of a continuous modification for the process $Y$ is given by the Proposition 7.2.

If, furthermore, $a \in C G I_{\gamma, \theta, q, T}$, then the stochastic integral may be represented as

$$
\int_{0}^{t} a(s) \mathrm{d} W_{K}(s)=\int_{0}^{t} I_{t}(a)(s) \mathrm{d} B_{s}+Z(t), \quad Z(t)=\int_{0}^{t} J_{t}(D .(a))(s) \mathrm{d} s .
$$

The process $Z$ is obviously a continuous process so that ends the proof.

Proposition 7.2. - Let $\beta>0$ such that $\beta+h_{\gamma}>\frac{1}{2}$. Assume that $a \in \tilde{\mathcal{H}}^{\beta}\left(\mathbf{D}^{1,2} \cap\right.$ $\left.\mathbf{L}^{1,2}\right)$ and $K \in A_{\gamma, \theta, p, T}^{\beta}$. Then the process $t \rightarrow \int_{0}^{t} J_{t}^{K}(a(\cdot, s))(s) \mathrm{d} B_{s}$ has a continuous modification.

In particular, if $a \in \mathbf{C}^{\beta} \mathbf{L}^{1,2}(0, T)$ then $\tilde{a}(u, s)=\Delta_{s, u}^{0} a=a(u)-a(s)$ is in $\tilde{\mathcal{H}}^{\beta}\left(\mathbf{D}^{1,2} \cap\right.$ $\left.\mathbf{L}^{1,2}\right)$ and $t \rightarrow \int_{0}^{t} J_{t}^{K}\left(\Delta^{0} a\right)(s) \mathrm{d} B_{s}$ has a continuous modification.

Proof. - According to Lemma 6.2(ii) we have $D_{r} J^{K}(a)(s)=J^{K}\left(D_{r} a\right)(s)$ and

$$
\left\|J_{t+\tau}^{K}(a(\cdot, s))(s)-J_{t}^{K}(a(\cdot, s))(s)\right\|_{\mathbf{L}^{1,2}} \leqslant C\|a\|_{\tilde{\mathcal{H}}^{\beta}\left(\mathbf{D}^{1,2}\right)}\|K\|_{A_{\gamma, \theta, p, T}^{\beta}} \tau^{\beta+h_{\gamma}} .
$$

Since $2\left(\beta+h_{\gamma}\right)>1$ we can conclude by Kolmogorov's continuity criterion.

\section{Itô's formula}

Itô's formula for fractional Brownian motion of Hurst parameter $H>\frac{1}{2}$ is now well known: see, for instance, Decreusefond and Ustunel [9], Dai and Heyde [8]. Here, we show how to obtain it for a wide family of kernels. The first step of our method is to write Itô's formula for a semi-martingale kernel in a suitable way, that is a way that will be easily extended to more general kernels.

PROPOSITION 8.1. - Let $K$ be a semi-martingale kernel and $f \in C_{b}^{2}$. Then, almost surely, for every $t>0$ :

$$
\begin{aligned}
f\left(W_{K}(t)\right)= & f(0)+\int_{0}^{t} I_{t}\left(f^{\prime}\left(W_{K}\right)\right)(s) \mathrm{d} B_{s} \\
& +\frac{1}{2} \int_{0}^{t} f^{\prime \prime}\left(W_{K}(s)\right) \frac{\mathrm{d}}{\mathrm{d} s} \mathbb{E}\left[W_{K}(s)^{2}\right] \mathrm{d} s .
\end{aligned}
$$

Proof. - We let $\phi(s)=\mathbb{E}\left[W_{K}(s)^{2}\right]=\int_{0}^{s} K(s, r)^{2} \mathrm{~d} r$. Since $K$ is a semi-martingale kernel, $\phi$ is differentiable with

$$
\phi^{\prime}(s)=K(s, s)^{2}+2 \int_{0}^{s} K(s, r) \partial_{1} K(s, r) \mathrm{d} r .
$$


By the chain rule,

$$
D_{s} f^{\prime}\left(W_{K}(u)\right)=f^{\prime \prime}\left(W_{K}(u)\right) D_{s}\left(W_{K}(u)\right)=f^{\prime \prime}\left(W_{K}(u)\right) K(u, s),
$$

and therefore the process $\left(f^{\prime}\left(W_{K}(s)\right) ; D \in[0, T]\right)$ is in $\mathbf{L}^{1,2}(0, t)$ and we may use Proposition 6.1. Furthermore,

$$
\begin{aligned}
\int_{0}^{t} J_{t}\left(D_{s} f^{\prime}\left(W_{K}\right)\right)(s) \mathrm{d} s & =\int_{0}^{t} \mathrm{~d} s \int_{s}^{t} \mathrm{~d} v \partial_{1} K(v, s) D_{s} f^{\prime}\left(W_{K}(v)\right) \\
& =\int_{0}^{t} \mathrm{~d} s \int_{s}^{t} \mathrm{~d} v \partial_{1} K(v, s) f^{\prime \prime}\left(W_{K}(v)\right) K(v, s) .
\end{aligned}
$$

Recall that the semi-martingale decomposition of $W_{K}$ is

$$
\mathrm{d} W_{K}(s)=K(s, s) \mathrm{d} B_{s}+W_{\partial_{1} K}(s) \mathrm{d} s .
$$

Therefore, Itô's formula yields

$$
\begin{aligned}
f\left(W_{K}(t)\right)= & f(0)+\int_{0}^{t} f^{\prime}\left(W_{K}(s)\right) \mathrm{d} W_{K}(s)+\frac{1}{2} \int_{0}^{t} f^{\prime \prime}\left(W_{K}(s)\right) K(s, s)^{2} \mathrm{~d} s \\
= & f(0)+\int_{0}^{t} I_{t}\left(f^{\prime}\left(W_{K}\right)\right)(s) \mathrm{d} B_{s}+\int_{0}^{t} J_{t}\left(D_{s} f^{\prime}\left(W_{K}\right)\right)(s) \mathrm{d} s \\
& +\frac{1}{2} \int_{0}^{t} f^{\prime \prime}\left(W_{K}(s)\right) K(s, s)^{2} \mathrm{~d} s \\
= & f(0)+\int_{0}^{t} I_{t}\left(f^{\prime}\left(W_{K}\right)\right)(s) \mathrm{d} B_{s}+\frac{1}{2} \int_{0}^{t} f^{\prime \prime}\left(W_{K}(s)\right) \phi^{\prime}(s) \mathrm{d} s .
\end{aligned}
$$

We shall make use of $B_{\gamma, \theta, p, T}^{\beta}$ defined in 2.6.

THEOREM 8.2. - Assume that for some $\frac{1}{2}-h_{\gamma}<\beta<h_{\gamma}$ the kernel $K$ is in $B_{\gamma, \theta, p, T}^{\beta}$ for every $T>0$, that $f \in C_{b}^{4}$, and that $h_{\gamma}>\frac{1}{4}$. Then, almost surely, for every $t>0$,

$$
\begin{aligned}
f\left(W_{K}(t)\right)= & f(0)+\int_{0}^{t} I_{t}\left(f^{\prime}\left(W_{K}\right)\right)(s) \mathrm{d} B_{s} \\
& +\frac{1}{2} \int_{0}^{t} f^{\prime \prime}\left(W_{K}(s)\right) \frac{\mathrm{d}}{\mathrm{d} s} \mathbb{E}\left[W_{K}(s)^{2}\right] \mathrm{d} s .
\end{aligned}
$$

Proof. - Our first step will be to prove that the processes on both sides have a continuous modification. Then, we shall establish this formula for fixed $t>0$. 
Since $W_{K}$ has a continuous modification, see Proposition 5.1, $f\left(W_{K}\right)$ also has a continuous modification. It is obvious that

$$
t \rightarrow f(0)+\frac{1}{2} \int_{0}^{t} f^{\prime \prime}\left(W_{K}(s)\right) \frac{\mathrm{d}}{\mathrm{d} s} \mathbb{E}\left[W_{K}(s)^{2}\right] \mathrm{d} s,
$$

also has a continuous modification.

In fact, Proposition 5.1 ensures that a.s. and in $L^{2}$, sample paths of the Gaussian process $W_{K}$ are Hölder continuous with index $\beta<h_{\gamma}$. Since $f^{\prime}, f^{\prime \prime}$ and $f^{(3)}$ are bounded, Proposition 8.4 ensures that $a=f^{\prime}\left(W_{K}\right)$ belongs to $\mathbf{C}^{\beta} \mathbf{L}^{1,2}(0, T) \cap L^{\infty}([0, T]$ $\times \Omega)$ as soon as $\beta<h_{\gamma}$. We furthermore impose that $\beta>\frac{1}{2}-h_{\gamma}>\gamma-1 / q$, which is possible since $h_{\gamma}>1 / 4$; then, according to Theorem 7.1, the process $t \rightarrow$ $\int_{0}^{t} I_{t}\left(f^{\prime}\left(W_{K}\right)\right)(s) \mathrm{d} B_{s}$ has a continuous modification.

Let us now establish formula (8.2) for a fixed $t>0$. Assume that $K_{n}$ is a family of semi-martingale kernels such that $K_{n} \rightarrow K$ in $B_{\gamma, \theta, p, T}^{\beta}$. Then formula (8.2) is valid for $K_{n}$.

We shall first handle the term

$$
\int_{0}^{t} f^{\prime \prime}\left(W_{K_{n}}(s)\right) \frac{\mathrm{d}}{\mathrm{d} s} \mathbb{E}\left[W_{K_{n}}(s)^{2}\right] \mathrm{d} s .
$$

According to the proof of Corollary 5.4, by taking a subsequence, we may assume that $\sup _{s \leqslant t}\left|W_{K_{n}}(s)-W_{K}(s)\right| \rightarrow 0$ both in $L^{2}$ and $\mathrm{n}$ almost surely. Therefore according to Lemma 2.13:

$$
\int_{0}^{t} f^{\prime \prime}\left(W_{K_{n}}(s)\right) \frac{\mathrm{d}}{\mathrm{d} s} \mathbb{E}\left[W_{K_{n}}(s)^{2}\right] \mathrm{d} s \rightarrow \int_{0}^{t} f^{\prime \prime}\left(W_{K}(s)\right) \frac{\mathrm{d}}{\mathrm{d} s} \mathbb{E}\left[W_{K}(s)^{2}\right] \mathrm{d} s .
$$

To prove the convergence

$$
\int_{0}^{t} I_{t} f^{\prime}\left(W_{K_{n}}\right)(s) \mathrm{d} B_{s} \rightarrow \int_{0}^{t} I_{t} f^{\prime}\left(W_{K}(s)\right) \mathrm{d} B_{s}
$$

we need to prove that $I_{t}^{K_{n}} a_{n} \rightarrow I_{t} a$ in $\mathbf{L}^{1,2}(0, t)$ where $a(s)=f^{\prime}\left(W_{K}(s)\right)$ and $a_{n}(s)=$ $f^{\prime}\left(W_{K_{n}}(s)\right)$. Thanks to Proposition 6.1, and to the fact that $K_{n} \rightarrow K$ in $B_{\gamma, \theta, p, T}^{\beta}$, it is enough to prove that $\left\|a-a_{n}\right\|_{\gamma, \theta, q, t} \underset{n \rightarrow \infty}{\longmapsto} 0$, and this we achieve in the following Proposition 8.4.

DEFINITION 8.3. - From now on, $\Delta^{0} F, \Delta^{1} F$ will denote the Taylor expansion of the function $F$ :

$$
\Delta_{x, y}^{0} F=F(y)-F(x), \quad \Delta_{x, y}^{1} F=F(y)-F(x)-(y-x) F^{\prime}(x) .
$$

Proposition 8.4. - Assume $f \in \mathcal{C}_{b}^{m+i+2}$ with $i=0,1$ and $m=0,1,2$. Then the mapping $K \rightarrow \Delta_{W_{K}(s), W_{K}(u)}^{i} f$ is continuous from $E_{\gamma, \theta, p, T}$ to $\mathcal{H}^{(i+1) h_{\gamma}}\left(\mathbf{D}^{m, 2}\right)$. 
Proof. - Let $X$ be a separable Hilbert space.

Definition 8.5. - A function $G: \mathbb{R}^{2} \times[0, T]^{2} \rightarrow X$ is said to satisfy assumption $\mathcal{H} \mathcal{H}(m, k, \tilde{k})$ if $G$ is $m$-times differentiable with respect to its first two variables, and if there exists a constant $C(G)=C(G, m, k, \tilde{k})$ such that for every real numbers $(x, y) \in \mathbb{R}^{2}, 0 \leqslant u, s \leqslant T$, integers $i, j$ such that $0 \leqslant i+j \leqslant m$, we have

$$
\left|\partial_{i, j}^{i+j} G(x, y, u, s)\right|_{X} \leqslant C(G) \sum_{l=0}^{j}|y|^{\max (k-l, 0)}|u-s|^{\tilde{k}} .
$$

Observe that if $f \in C_{b}^{M+m}$, then $G(x, y, u, s)=\Delta_{x, x+y}^{M-1} f=\frac{1}{M !} \int_{0}^{1} f^{(M)}(x+\theta y) y^{M} \mathrm{~d} \theta$ satisfies $\mathcal{H} \mathcal{H}(m, M, 0)$. Therefore Proposition 8.4 is an immediate consequence of the following lemma.

Lemma 8.6. - Assume $\alpha \geqslant 1$ and $G$ satisfies $\mathcal{H} \mathcal{H}(m, k ; \tilde{k})$. Then for every $K \in$ $E_{\gamma, \theta, p, T}, 0 \leqslant u, s \leqslant T$,

$$
\left\|G\left(W_{K}(s), \Delta_{s, u}^{0} W_{K}, u, s\right)\right\|_{\mathbf{D}^{n, \alpha}(X)} \leqslant C \times C(G) \times \sum_{j=0}^{m}\|K\|_{\gamma, \theta, p, T}^{k+j} \times|u-s|^{k h_{\gamma}+\tilde{k}} .
$$

Proof. - The proof is an induction on the integer $m$.

Assume that $G$ satisfies $\mathcal{H} \mathcal{H}(0, k, \tilde{k})$. Then,

$$
\left|G\left(W_{K}(s) ; \Delta_{s, u}^{0} W_{K} ; u, s\right)\right|_{X} \leqslant C(G)\left|\Delta_{s, u}^{0} W_{K}\right|^{k}|u-s|^{\tilde{k}} .
$$

Since the random variable $\Delta_{s, u}^{0} W_{K}$ is centered with covariance $\left\|\Delta_{s, u}^{0} K(\cdot, r)\right\|_{L^{2}([0, T], \mathrm{d} r)}$ with the convention $K(u, r)=0$ for $u<r$, we deduce from Proposition 2.10 the upper bound

$$
\left\|\Delta_{s, u}^{0} K(\cdot, r)\right\|_{L^{2}([0, T], \mathrm{d} r)} \leqslant C\|K\|_{\gamma, \theta, p, T}|u-s|^{h_{\gamma}} .
$$

We plug in the expression of the $k \alpha$ th order moment of a centered Gaussian random variable, to obtain that the lemma is satisfied for a constant

$$
C^{\prime}=C\|\mathcal{N}(0,1)\|_{k h_{\gamma}}^{k} .
$$

We now assume the lemma satisfied for $m$ and let $G$ verify assumption $\mathcal{H} \mathcal{H}(m+1, k, \tilde{k})$. Then $G$ is differentiable with respect to the first two variables up to the order $m+1$, and $G$ together with its partial derivatives have at most polynomial growth. According to the chain rules of Malliavin Calculus, the random variable $G\left(W_{K}(s), \Delta_{s, u}^{0} W_{K} ; u, s\right)$ belongs to $\mathbf{D}^{m+1, \alpha}$ and

$$
D^{m+1}\left[G\left(W_{K}(s), \Delta_{s, u}^{0} W_{K} ; u, s\right)\right]=\sum_{\sigma \in \mathcal{S}_{m+1}, i+j=m+1} G^{i, j, \sigma}\left(W_{K}(s), \Delta_{s, u}^{0} W_{K} ; u, s\right),
$$

where $\mathcal{S}_{n}$ denotes the group of order $n$ permutations, $\otimes$ the tensor product and 


$$
\begin{aligned}
& G(i, j, \sigma)(x, y, u, s)\left(r_{1}, \ldots, r_{m+1}\right) \\
& \quad=\frac{1}{i ! j !} \partial_{i, j}^{i+j} G(x, y, u, s) \otimes^{i} K(s, \cdot)\left(r_{\sigma(1)}, \ldots, r_{\sigma(i)}\right) \\
& \quad \otimes^{j} \Delta_{s, u}^{0} K(\cdot, \cdot)\left(r_{\sigma(i+1)}, \ldots, r_{\sigma(m+1)}\right) .
\end{aligned}
$$

Injecting the upper bound 8.3, we get that $G(i, j, \sigma)$ satisfies $\mathcal{H} \mathcal{H}\left(0, k-j, \tilde{k}+j h_{\gamma}\right)$ on $X=L^{2}\left([0, T]^{m+1}\right)$. We can therefore apply the lemma for $m=0$ to every $G(i, j, \sigma)$ to obtain

$$
\begin{aligned}
& \left\|D^{m+1}\left[G\left(W_{K}(s), \Delta_{s, u}^{0} W_{K} ; u, s\right)\right]\right\|_{L^{\alpha}(X)} \\
& \quad \leqslant C C(G, m+1) \sum_{j}\|K\|_{\gamma, \theta, p, T}^{k+j}|u-s|^{k h_{\gamma}+\tilde{k}} .
\end{aligned}
$$

We conclude by using the definition of the norm in $\mathbf{D}^{\alpha, m+1}$ and the induction assumption.

LEMmA 8.7. - Assume that $G$ satisfies $\mathcal{H} \mathcal{H}(m, k, \tilde{k})$ with $m \geqslant 1, k>0$. Then the mapping $K \rightarrow G\left(W_{K}(s), \Delta_{s, u}^{0} W_{K}, u, s\right)$ is continuous from $E_{\gamma, \theta, p, T}$ to $\tilde{\mathcal{H}}^{k h_{\gamma}+\tilde{k}}\left(\mathbf{D}^{m-1,2}\right) \cap$ $\mathbf{L}^{1,2}$ (with the convention $\mathbf{L}^{0,2}=L^{2}(\Omega)$ ).

Proof. - Using Taylor integral expansion we have for $K, K^{\prime} \in E_{\gamma, \theta, p, T}$

$$
G\left(W_{K}(s) ; \Delta_{s, u}^{0} W_{K} ; u ; s\right)-G\left(W_{K^{\prime}}(s) ; \Delta_{s, u}^{0} W_{K^{\prime}} ; u ; s\right)=\int_{0}^{1} B_{\theta} \mathrm{d} \theta
$$

with

$$
B_{\theta}=\nabla G\left(W_{K+\theta\left(K-K^{\prime}\right)}(s) ; \Delta_{s, u}^{0} W_{K+\theta\left(K-K^{\prime}\right)} ; u, s\right)\left[W_{K-K^{\prime}}(s) ; \Delta_{u, s}^{0} W_{K-K^{\prime}}\right]^{t},
$$

where $x^{t}$ denotes the transpose of $x$.

For $i=1,2$, the mapping $\partial_{i} G(x, y, u, s)$ satisfies $\mathcal{H} \mathcal{H}(m-1, k+1-i, \tilde{k})$. Therefore Lemma 8.6 implies

$$
\begin{aligned}
& \left\|\partial_{i} G\left(W_{K+\theta\left(K-K^{\prime}\right)} ; \Delta_{s, u}^{0} W_{K+\theta\left(K-K^{\prime}\right)} ; s, u\right)\right\|_{\mathbf{D}^{m-1,2}} \\
& \quad \leqslant C|u-s|^{(k+1-i) h_{\gamma}+\tilde{k}} \sum_{j=0}^{m-1}\left(\|K\|_{\gamma, \theta, p, T}^{k+j}+\left\|K^{\prime}\right\|_{\gamma, \theta, p, T}^{k+j}\right) .
\end{aligned}
$$

The same Lemma 8.6 yields

$$
\begin{aligned}
& \left\|W_{K-K^{\prime}}(s)\right\|_{\mathbf{D}^{m-1,2}} \leqslant C\left\|K-K^{\prime}\right\|_{\gamma, \theta, p, T}, \\
& \left\|\Delta_{s, u}^{0} W_{K-K^{\prime}}\right\|_{\mathbf{D}^{m-1,2}} \leqslant C\left\|K-K^{\prime}\right\|_{\gamma, \theta, p, T}|u-s|^{h_{\gamma}} .
\end{aligned}
$$

Combining (8.7) and (8.8) yields,

$$
\left\|B_{\theta}\right\|_{\mathbf{D}^{m-1,2}} \leqslant C \sum_{j=1}^{m-1}\left(\|K\|_{\gamma, \theta, p, T}^{k+j}+\left\|K^{\prime}\right\|_{\gamma, \theta, p, T}^{k+j}\right)\left\|K-K^{\prime}\right\||u-s|^{k h_{\gamma}+\tilde{k}}
$$

and injecting this into (8.6) yields the desired result. 


\subsection{Application to fractional Brownian motion}

According to Lemma 2.7 we can take $h_{\gamma}=H-\varepsilon$ with $\varepsilon>0$ small enough so that $h_{\gamma}>\frac{1}{4}$ and then use Theorem 8.2.

\subsection{Ito formula for $H>\frac{1}{6}$}

We first derive a new expression for the Ito formula for the Gaussian processes associated to semi-martingale kernels.

Proposition 8.8. - Let $K$ a semi-martingale kernel and $f \in C_{b}^{4}$. Then, for $t \in$ $[0, T]$ almost everywhere,

$$
\begin{aligned}
f\left(W_{K}(t)\right)= & f(0)+\frac{1}{2} \int_{0}^{t} f^{\prime \prime}\left(W_{K}(s)\right) \frac{\mathrm{d}}{\mathrm{d} s} R_{K}(s, s) \mathrm{d} s \\
& +\int_{0}^{t} K(t, s)\left(f^{\prime}\left(W_{K}(s)\right)+f^{\prime \prime}\left(W_{K}(s)\right) \mathbb{E}\left[W_{K}(t)-W_{K}(s) \mid \mathcal{F}_{s}\right]\right) \mathrm{d} B_{s} \\
& -\int_{0}^{t} K(t, s) f^{\prime \prime \prime}\left(W_{K}(s)\right) \mathbb{E}\left[\left(W_{K}(t)-W_{K}(s)\right) W_{K}(s)\right] \mathrm{d} B_{s} \\
& +\int_{0}^{t} I_{t}^{K}\left[\Delta_{W_{K}(s), W_{K}(\cdot)}^{1} f^{\prime}+f^{\prime \prime \prime}\left(W_{K}(s)\right) \mathbb{E}\left[\left(W_{K}(\cdot)-W_{K}(s)\right) W_{K}(s)\right]\right](s) \mathrm{d} B_{s} \\
& -\int_{0}^{t}\left(\int _ { 0 } ^ { s } \left[\left(f^{\prime \prime}\left(W_{K}(s)\right)-f^{\prime \prime}\left(W_{K}\left(s_{1}\right)\right)\right)\right.\right. \\
& \left.\left.\times \int_{s}^{t} \partial_{1} K\left(u, s_{1}\right) K(u, s) \mathrm{d} u\right] \mathrm{~d} B_{s_{1}}\right) \mathrm{d} B_{s}
\end{aligned}
$$

with $R_{K}$ the covariance function of the Gaussian process $W_{K}$

$$
R_{K}(x, y)=\mathbb{E}\left[W_{K}(x) W_{K}(y)\right] .
$$

Proof. - Let $K$ a semi-martingale kernel and $f \in C_{b}^{4}$. According to Proposition 8.1, for $t \in[0, T]$, we have

$$
\begin{aligned}
f\left(W_{K}(t)\right)= & f(0)+\int_{0}^{t} I_{t}^{K}\left(f^{\prime}\left(W_{K}\right)\right)(s) \mathrm{d} B(s) \\
& +\frac{1}{2} \int_{0}^{t} f^{\prime \prime}\left(W_{K}(s)\right) \frac{\mathrm{d}}{\mathrm{d} s} \mathbb{E}\left[W_{K}(s)^{2}\right] \mathrm{d} s .
\end{aligned}
$$

A Taylor expansion of $f^{\prime}$ between $W_{K}(u)$ and $W_{K}(s)$ yields 


$$
\begin{aligned}
I_{t}^{K}\left(f^{\prime}\left(W_{K}\right)\right)(s)= & K(t, s) f^{\prime}\left(W_{K}(s)\right)+I_{t}^{K}\left(\Delta_{W_{K}(s), W_{K}(\cdot)}^{1} f^{\prime}\right)(s) \\
& +f^{\prime \prime}\left(W_{K}(s)\right) I_{t}^{K}\left(\Delta_{s, \cdot}^{0} W_{K}\right)(s) .
\end{aligned}
$$

Since $f \in C_{b}^{4}$, the process $\left(a(s)=f^{\prime \prime}\left(W_{K}(s)\right), 0 \leqslant s \leqslant T\right)$ belongs to $\mathbf{L}^{2,2}$ and

$$
\begin{aligned}
& \int_{0}^{s} D_{s_{1}} a(s) \int_{s}^{t} K(u, s) \partial_{1} K\left(u, s_{1}\right) \mathrm{d} u \mathrm{~d} s_{1} \\
& \quad=f^{\prime \prime \prime}\left(W_{K}(s)\right) \int_{s}^{t} K(u, s) \frac{\mathrm{d}}{\mathrm{d} s} \mathbb{E}\left[W_{K}(u) W_{K}(s)\right] \mathrm{d} u .
\end{aligned}
$$

Using the integration by parts formula,

$$
\begin{aligned}
& \int_{0}^{s} D_{s_{1}} a(s) \int_{s}^{t} K(u, s) \partial_{1} K\left(u, s_{1}\right) \mathrm{d} u \mathrm{~d} s_{1} \\
& \quad=f^{\prime \prime \prime}\left(W_{K}(s)\right)\left(K(t, s) \mathbb{E}\left[\Delta_{s, t}^{0} W_{K} W_{K}(s)\right]-J_{t}^{K}\left(\mathbb{E}\left[\Delta_{s, \cdot}^{0} W_{K} W_{K}(s)\right]\right)(s)\right) .
\end{aligned}
$$

Then Lemma 8.9 yields,

$$
\begin{aligned}
f\left(W_{K}(t)\right)= & f(0)+\frac{1}{2} \int_{0}^{t} f^{\prime \prime}\left(W_{K}(s)\right) \frac{\mathrm{d}}{\mathrm{d} s} R_{K}(s, s) \mathrm{d} s \\
& +\int_{0}^{t}\left[f^{\prime}\left(W_{K}(s)\right)+f^{\prime \prime}\left(W_{K}(s)\right) \mathbb{E}\left[\Delta_{s, t}^{0} W_{K} \mid \mathcal{F}_{s}\right]\right] K(t, s) \mathrm{d} B_{s} \\
& +\int_{0}^{t}\left[f^{\prime \prime \prime}\left(W_{K}(s)\right) \mathbb{E}\left[\Delta_{s, t}^{0} W_{K} W_{K}(s)\right]\right] K(t, s) \mathrm{d} B_{s} \\
& +\int_{0}^{t} I_{t}^{K}\left[\Delta_{W_{K}(s), W_{K}(\cdot)}^{1} f^{\prime}+f^{\prime \prime \prime}\left(W_{K}(s)\right) \mathbb{E}\left[\Delta_{s, \cdot}^{0} W_{K} W_{K}(s)\right]\right](s) \mathrm{d} B_{s} \\
& +\int_{0}^{t}\left(\int_{0}^{s} \Delta_{W_{K}(s), W_{K}\left(s_{1}\right)}^{0} f^{\prime \prime} J_{t}^{K}(K(\cdot, s))\left(s_{1}\right) \mathrm{d} B_{s_{1}}\right) \mathrm{d} B_{s} .
\end{aligned}
$$

LEMMA 8.9. - Let $K$ be a semi-martingale kernel and $a \in \mathbf{L}^{2,2}$ an adapted process. Then

$$
\begin{aligned}
& \int_{0}^{t} a(s) I_{t}^{K}\left(\Delta_{s, \cdot}^{0}, W_{K}\right)(s) \mathrm{d} B_{s} \\
& \quad=\int_{0}^{t} a(s) \mathbb{E}\left[\Delta_{s, t}^{0} W_{K} \mid \mathcal{F}_{s}\right] K(t, s) \mathrm{d} B_{s}
\end{aligned}
$$




$$
\begin{aligned}
& +\int_{0}^{t}\left[\int_{0}^{s}\left(\Delta_{s, s_{1}}^{0} a \int_{s}^{t} K(u, s) \partial_{1} K\left(u, s_{1}\right) \mathrm{d} u\right) \mathrm{d} B_{s_{1}}\right] \mathrm{d} B_{s} \\
& -\int_{0}^{t}\left(\int_{0}^{s}\left[D_{s_{1}} a(s) \int_{0}^{t} K(u, s) \partial_{1} K\left(u, s_{1}\right) \mathrm{d} u\right] \mathrm{d} s_{1}\right) \mathrm{d} B_{s} .
\end{aligned}
$$

Proof. - We split $W_{K}(u)-W_{K}(s)=\Delta_{s, u}^{0} W_{K}$ in two parts:

$$
\Delta_{s, u}^{0} W_{K}=\mathbb{E}\left[\Delta_{s, u}^{0} W_{K} \mid \mathcal{F}_{s}\right]+\left(\Delta_{s, u}^{0} W_{K}-\mathbb{E}\left[\Delta_{s, u}^{0} W_{K} \mid \mathcal{F}_{s}\right]\right)
$$

Observe that $W_{K_{E}}=\left(\mathbb{E}\left[\Delta_{s, t}^{0} W_{K} \mid \mathcal{F}_{s}\right], s \leqslant t \leqslant T\right)$ is a centered Gaussian process that can be written, thanks to Fubini's Stochastic Theorem,

$$
W_{K_{E}}(t)=\int_{0}^{s} \Delta_{s, t}^{0} K\left(\cdot, s_{1}\right) \mathrm{d} B_{s_{1}}=\int_{s}^{t} \mathrm{~d} u\left(\int_{0}^{u \wedge s} \partial_{1} K\left(u, s_{1}\right) \mathrm{d} B_{s_{1}}\right) .
$$

The integration by parts formula (applied to the semi-martingale $W_{K_{E}}$ and the deterministic kernel $K(\cdot, s))$ yields

$$
\begin{aligned}
K(t, s) W_{K_{E}}(t)= & K(s, s) W_{K_{E}}(s)+\int_{s}^{t} W_{K_{E}}(u) \partial_{1} K(u, s) \mathrm{d} u \\
& +\int_{s}^{t} K(u, s) \mathrm{d} W_{K_{E}}(u) .
\end{aligned}
$$

Since $a$ is adapted and $W_{K_{E}}(s)=0$, we obtain

$$
\begin{aligned}
& \int_{s}^{t} a(s) \mathbb{E}\left[\Delta_{s, u}^{0} W_{K} \mid \mathcal{F}_{s}\right] \partial_{1} K(u, s) \mathrm{d} u \\
& \quad=a(s) \mathbb{E}\left[\Delta_{s, t}^{0} W_{K} \mid \mathcal{F}_{s}\right] K(t, s)-\int_{0}^{t} a(s) 1_{(s, t)}(u) K(u, s) \mathrm{d} W_{K_{E}}(u) \\
& =a(s) \mathbb{E}\left[\Delta_{s, t}^{0} W_{K} \mid \mathcal{F}_{s}\right] K(t, s)-\int_{s}^{t} a(s) K(u, s)\left(\int_{0}^{s} \partial_{1} K\left(u, s_{1}\right) \mathrm{d} B_{s_{1}}\right) \mathrm{d} u .
\end{aligned}
$$

The integration by parts formula yields then, as in the proof of Proposition 6.1

$$
\begin{aligned}
\int_{s}^{t} a(s) \mathbb{E}\left[\Delta_{s, u}^{0} W_{K} \mid \mathcal{F}_{s}\right] \partial_{1} K(u, s) \mathrm{d} u \\
=a(s) \mathbb{E}\left[W_{K}(t)-W_{K}(s) \mid \mathcal{F}_{s}\right] K(t, s) \\
\quad-\int_{0}^{s}\left(a(s) \int_{s}^{t} K(u, s) \partial_{1} K\left(u, s_{1}\right) \mathrm{d} u\right) \mathrm{d} B_{s_{1}}
\end{aligned}
$$




$$
-\int_{0}^{s}\left(\int_{s}^{t} D_{s_{1}} a(s) K(u, s) \partial_{1} K\left(u, s_{1}\right) \mathrm{d} u\right) \mathrm{d} s_{1} .
$$

Integrating with respect to $\mathrm{d} B(s)$ each term of this equality we obtain,

$$
\begin{gathered}
\int_{0}^{t}\left(a(s) \int_{s}^{t} \mathbb{E}\left[W_{K}(u)-W_{K}(s) \mid \mathcal{F}_{s}\right] \partial_{1} K(u, s) \mathrm{d} u\right) \mathrm{d} B_{s} \\
=\int_{0}^{t} a(s) \mathbb{E}\left[W_{K}(t)-W_{K}(s) \mid \mathcal{F}_{s}\right] K(t, s) \mathrm{d} B_{s} \\
-\int_{0}^{t}\left[\int_{0}^{s}\left(a(s) \int_{s}^{t} K(u, s) \partial_{1} K\left(u, s_{1}\right) \mathrm{d} u\right) \mathrm{d} B_{s_{1}}\right] \mathrm{d} B_{s} \\
-\int_{0}^{t}\left[\int_{0}^{s}\left(\int_{s}^{t} D_{s_{1}} a(s) K(u, s) \partial_{1} K\left(u, s_{1}\right) \mathrm{d} u\right) \mathrm{d} s_{1}\right] \mathrm{d} B_{s} .
\end{gathered}
$$

Observe that for $s \leqslant t$,

$$
W_{K}(u)-W_{K}(s)-\mathbb{E}\left[W_{K}(u)-W_{K}(s) \mid \mathcal{F}_{s}\right]=\int_{s}^{t} K\left(u, s_{1}\right) \mathrm{d} B\left(s_{1}\right)
$$

and using again stochastic integration by parts and Fubini's Stochastic Theorem for deterministic integrands, we obtain that

$$
\begin{gathered}
\int_{0}^{t} \int_{s}^{t} a(s)\left(W_{K}(u)-W_{K}(s)-\mathbb{E}\left[W_{K}(u)-W_{K}(s) \mid \mathcal{F}_{s}\right]\right) \partial_{1} K(u, s) \mathrm{d} u \mathrm{~d} B_{s} \\
=\int_{0}^{t}\left(\int_{0}^{s_{1}} a(s) \int_{s}^{t} K\left(u, s_{1}\right) \partial_{1} K(u, s) \mathrm{d} u \mathrm{~d} B_{s}\right) \mathrm{d} B_{s_{1}} .
\end{gathered}
$$

Summing up this equality with (8.9) yields, after one substitution $s \leftrightarrow s_{1}$,

$$
\begin{aligned}
& \int_{0}^{t} a(s) I_{t}^{K}\left(W_{K}(\cdot)-W_{K}(s)\right)(s) \mathrm{d} B_{s} \\
& =\int a(s) \mathbb{E}\left[W_{K}(t)-W_{K}(s) \mid \mathcal{F}_{s}\right] K(t, s) \mathrm{d} B_{s} \\
& \quad-\int_{0}^{t}\left[\int_{0}^{s}\left(\left(a(s)-a\left(s_{1}\right)\right) \int_{s}^{t} K(u, s) \partial_{1} K\left(u, s_{1}\right) \mathrm{d} u\right) \mathrm{d} B_{s_{1}}\right] \mathrm{d} B_{s} \\
& \quad-\int_{0}^{t}\left(\int _ { 0 } ^ { s } \left[\begin{array}{c}
\left.\left.D_{s_{1}} a(s) \int_{0}^{t} K(u, s) \partial_{1} K\left(u, s_{1}\right) \mathrm{d} u\right] \mathrm{~d} s_{1}\right) \mathrm{d} B_{s} .
\end{array}\right.\right.
\end{aligned}
$$


Proposition 8.10. - Assume $\beta+2 h_{\gamma}>\frac{1}{2}$. Then the mapping

$$
\begin{aligned}
(K, & \left.K^{\prime}, a\right) \rightarrow a\left(s, s_{1}\right) J_{t}^{K}\left(K^{\prime}(\cdot, s)\right)\left(s_{1}\right) 1_{\left(s_{1}<s\right)} \\
& =a\left(s, s_{1}\right) 1_{\left(s_{1}<s\right)} \int_{s}^{t} K^{\prime}(u, s) \partial_{1} K\left(u, s_{1}\right) \mathrm{d} u
\end{aligned}
$$

is multilinear continuous from $A_{\gamma, \theta, p, T}^{\beta} \times E_{\gamma, \theta, p, T} \times\left(L_{w_{\beta}}^{\infty}\left(\mathbf{D}^{2,2}\right) \cap \mathbf{L}^{2,2}\right)$ to $\mathbf{L}^{2,2}$. In particular, there exists a constant $C$ such that

$$
\begin{aligned}
& \left\|\int_{0}^{t} \mathrm{~d} B(s) \int_{0}^{s}\left(a\left(s, s_{1}\right) J_{t}^{K}\left(K^{\prime}(\cdot, s)\right)\left(s_{1}\right)\right) \mathrm{d} B\left(s_{1}\right)\right\|_{L^{2}} \\
& \quad \leqslant C\|a\|_{\tilde{\mathcal{H}}^{\beta}}\|K\|_{A_{\gamma, \theta, p, T}^{\beta}}\left\|K^{\prime}\right\|_{\gamma, \theta, p, T} .
\end{aligned}
$$

Moreover, the process

$$
t \rightarrow \int_{0}^{t} \mathrm{~d} B(s) \int_{0}^{s}\left(a\left(s, s_{1}\right) \int_{s}^{t} K^{\prime}(u, s) \partial_{1} K(u, s) \mathrm{d} u\right) \mathrm{d} B_{s_{1}}
$$

has a continuous modification on $[0, T]$.

Proof. - According to the definition (2.17) of $J_{2, t}$ we have

$$
a\left(s, s_{1}\right) J_{t}^{K}\left(K^{\prime}(\cdot, s)\right)\left(s_{1}\right) 1_{\left(s_{1}<s\right)}=J_{2, t}^{K, K^{\prime}}(a)\left(s, s_{1}\right) .
$$

Hence, Lemma 2.14 implies that

$$
\left\|J_{2, t}^{K^{\prime}, K}(a)\left(s, s_{1}\right)\right\|_{\mathbf{L}^{2,2}} \leqslant C\|a\|_{\tilde{\mathcal{H}}^{\beta}}\|K\|_{A_{\gamma, \theta, p, T}^{\beta}}\left\|K^{\prime}\right\|_{\gamma, \theta, p, T} .
$$

Moreover, thanks to the same Lemma 2.14, if

$$
X(t)=\int_{0}^{t} \mathrm{~d} B(s) \int_{0}^{s}\left(a\left(s, s_{1}\right) J^{K}\left(K^{\prime}(\cdot, s)\right)(s) 1_{\left(s_{1}<s\right)}\right) \mathrm{d} B_{s_{1}}
$$

then we have, for $0 \leqslant t \leqslant t+\tau \leqslant T$,

$$
\|X(t+\tau)-X(t)\|_{L^{2}} \leqslant C\|a\|_{\tilde{\mathcal{H}}^{\beta}}\|K\|_{A_{\gamma, \theta, p, T}^{\beta}}\left\|K^{\prime}\right\|_{\gamma, \theta, p, T} \tau^{\beta+2 h_{\gamma}} .
$$

Since $2\left(\beta+2 h_{\gamma}\right)>1$, Kolmogorov's continuity criterion ensures the existence of a continuous modification.

We can now state and prove an Ito formula that applies to the family of Gaussian processes $W_{K}$ such that $h_{\gamma}>\frac{1}{6}$ and therefore, by Lemma 2.7 to fractional Brownian motion with Hurst index $H>\frac{1}{6}$. 
Proposition 8.11. - Let $K \in B_{\gamma, \theta, p, T}^{\beta}$ with $\beta / 2>h_{\gamma}>\frac{1}{6}$ and let $f \in \mathcal{C}_{b}^{6}$. Then almost surely, for $0<t \leqslant T$,

$$
\begin{aligned}
f\left(W_{K}(t)\right)= & f(0)+\frac{1}{2} \int_{0}^{t} f^{\prime \prime}\left(W_{K}(s)\right) \frac{\mathrm{d}}{\mathrm{d} s} \mathbb{E}\left[W_{K}(s)^{2}\right] \mathrm{d} s \\
& +\int_{0}^{t}\left[f^{\prime}\left(W_{K}(s)\right)+f^{\prime \prime}\left(W_{K}(s)\right) \mathbb{E}\left[W_{K}(t)-W_{K}(s) \mid \mathcal{F}_{s}\right]\right] K(t, s) \mathrm{d} B(s) \\
& -\int_{0}^{t}\left[f^{\prime \prime \prime}\left(W_{K}(s)\right) \mathbb{E}\left[\left(W_{K}(t)-W_{K}(s)\right) W_{K}(s)\right]\right] K(t, s) \mathrm{d} B(s) \\
& +\int_{0}^{t} I_{t}^{K}\left(\Delta_{W_{K}(s), W_{K}(\cdot)}^{1} f^{\prime}+f^{\prime \prime \prime}\left(W_{K}(s)\right) \mathbb{E}\left[\left(W_{K}(\cdot)-W_{K}(s)\right) W_{K}(s)\right]\right)(s) \mathrm{d} B_{s} \\
& +\int_{0}^{t} \mathrm{~d} B_{s} \int_{0}^{s} \Delta_{s, s_{1}}^{0} f^{\prime \prime}\left(W_{K}\right) I_{t}^{K}(K(\cdot, s))\left(s_{1}\right) \mathrm{d} B_{s_{1}} .
\end{aligned}
$$

Proof. - The first step of the proof consists in showing that every process involved in this formula has a continuous modification. Then we shall prove in a second step that the formula is valid for each fixed $t$ by establishing the continuity with respect to $K \in F_{\gamma, \theta, p, T}$ for the norm $\|\cdot\|_{\gamma, \theta, p, T}$ and using Proposition 8.8 . We conclude by using the density of $F_{\gamma, \theta, p, T}$ into $B_{\gamma, \theta, p, T}^{\beta}$.

\section{Step 1: Existence of a continuous modification}

(1) Since $f^{(4)}$ is bounded, Proposition 8.4 entails that $\Delta_{W_{K}(s), W_{K}(u)}^{1} f^{\prime}$ belongs to $\tilde{\mathcal{H}}^{2 h_{\gamma}}\left(\mathbf{D}^{1,2}\right)$. Hence, we infer from Proposition 7.2 that since $3 h_{\gamma}>\frac{1}{2}$, the process

$$
t \rightarrow \int_{0}^{t} I_{t}^{K}\left(\Delta_{W_{K}(s), W_{K}(\cdot)}^{1} f^{\prime}\right)(s) \mathrm{d} B_{s}
$$

has a continuous modification.

(2) Similarly, since $f \in C_{b}^{4}$, Lemma 2.15 implies that we may apply Proposition 7.2 to the process $\left(f^{\prime \prime \prime}\left(W_{K}(s)\right) \mathbb{E}\left[\left(W_{K}(u)-W_{K}(s)\right) W_{K}(s)\right] ; 0 \leqslant u, s \leqslant T\right)$ to obtain the existence of a continuous modification for

$$
t \rightarrow \int_{0}^{t} I_{t}^{K}\left(f^{\prime \prime \prime}\left(W_{K}(s)\right) \mathbb{E}\left[\left(W_{K}(u)-W_{K}(s)\right) W_{K}(s)\right]\right)(s) \mathrm{d} B_{s} .
$$


(3) Since $f \in C_{b}^{5}$ we can apply Proposition 8.10 to $\Delta_{W_{K}(s), W_{K}\left(s_{1}\right)}^{0} f^{\prime \prime}$ to obtain the existence of a continuous modification for

$$
t \rightarrow \int_{0}^{t} \mathrm{~d} B_{s} \int_{0}^{s} \Delta_{s, s_{1}}^{0} f^{\prime \prime}\left(W_{K}\right) I_{t}^{K}(K(\cdot, s))\left(s_{1}\right) \mathrm{d} B_{s_{1}} .
$$

(4) The existence of a continuous modification for

$$
t \rightarrow \int_{0}^{t} f^{\prime \prime}\left(W_{K}(s)\right) \mathbb{E}\left[W_{K}(t)-W_{K}(s) \mid \mathcal{F}_{s}\right] K(t, s) \mathrm{d} B_{s}
$$

is a consequence of Burkholder-Davis-Gundy inequalities, Proposition 5.1, and the fact that $f^{\prime \prime}$ is bounded.

(5) Eventually, since $f \in C_{b}^{3}$ the existence of a continuous modification for

$$
t \rightarrow \int_{0}^{t} f^{\prime \prime \prime}\left(W_{K}(s)\right) \mathbb{E}\left[W_{K}(s)\left(W_{K}(t)-W_{K}(s)\right)\right] K(t, s) \mathrm{d} B_{s}
$$

is a consequence of Burkholder-Davis-Gundy inequalities and Lemma 2.15.

\section{Step 2: Itô's formula for a fixed time}

According to the definition 2.6, of $B_{\gamma, \theta, p, T}^{\beta}$, the mapping $K \rightarrow \int_{0}^{t} f^{\prime \prime}\left(W_{K}(s)\right) \times$ $\frac{\mathrm{d}}{\mathrm{d} s} \mathbb{E}\left[W_{K}(s)^{2}\right] \mathrm{d} s$ is continuous on $B_{\gamma, \theta, p, T}^{\beta}$.

In order to establish the continuity of the linear application

$$
\begin{aligned}
K \rightarrow & \int_{0}^{t}\left[f^{\prime}\left(W_{K}(s)\right)+f^{\prime \prime}\left(W_{K}(s)\right) \mathbb{E}\left[W_{K}(t)-W_{K}(s) \mid \mathcal{F}_{s}\right]\right] K(t, s) \mathrm{d} B(s) \\
& -\int_{0}^{t}\left[f^{\prime \prime \prime}\left(W_{K}(s)\right) \mathbb{E}\left[\left(W_{K}(t)-W_{K}(s)\right) W_{K}(s)\right]\right] K(t, s) \mathrm{d} B(s)
\end{aligned}
$$

we only need to prove the continuity from $E_{\gamma, \theta, p, T}$ into the space $L^{\alpha}$, for some $\alpha \geqslant \theta^{*} / 2$, of the integrand mapping

$$
\begin{aligned}
K \rightarrow & f^{\prime}\left(W_{K}(s)\right)+f^{\prime \prime}\left(W_{K}(s)\right) \mathbb{E}\left[W_{K}(t)-W_{K}(s) \mid \mathcal{F}_{s}\right] \\
& -f^{\prime \prime \prime}\left(W_{K}(s)\right) \mathbb{E}\left[\left(W_{K}(t)-W_{K}(s)\right) W_{K}(s)\right] .
\end{aligned}
$$

This can be obtained by Taylor expansions for $f^{\prime}, f^{\prime \prime}$ and $f^{\prime \prime \prime}$, Proposition 5.1, Lemma 2.15 and the following upper bound, proved for any adapted process $b$ bounded in $L^{\theta^{*} / 2}$ via Burkholder-Davis-Gundy and Hölder $\left(\theta / 2, \theta^{*} / 2\right)$ inequalities

$$
\left\|\int_{0}^{t} K(t, s) b(s) \mathrm{d} B_{s}\right\|_{L^{2}} \leqslant C\|K\|_{\gamma, \theta, p, T}\left(\int_{0}^{T} \mathbb{E}\left[|b(s)|^{\theta^{*} / 2}\right] \mathrm{d} s\right)^{1 / \theta^{*}} .
$$


Thanks to Lemma 6.2, the continuity of

$$
K \rightarrow \int_{0}^{t} I_{t}^{K}\left(\Delta_{W_{K}(s), W_{K}(\cdot)}^{1} f^{\prime}\right)(s) \mathrm{d} B_{s}
$$

is a consequence of the continuity of

$$
K \in E_{\gamma, \theta, p, T} \rightarrow \Delta_{W_{K}(s), W_{K}(u)}^{1} f^{\prime} \in \tilde{\mathcal{H}}^{\beta}\left(\mathbf{D}^{2,2}\right)
$$

for some $\beta>h_{\gamma}-\frac{1}{q}$. This latter continuity is an easy consequence of Proposition 8.4 applied to $\beta=2 h_{\gamma}$.

Since $h_{\gamma}>1 / 6$, combining Lemmas 6.2 and 2.15 with a Taylor expansion of $f^{\prime \prime \prime}$, shows that the mapping

$$
K \rightarrow \int_{0}^{t} I_{t}^{K}\left(f^{\prime \prime \prime}\left(W_{K}(s)\right) \mathbb{E}\left[\left(W_{K}(\cdot)-W_{K}(s)\right) W_{K}(s)\right]\right)(s) \mathrm{d} B_{s}
$$

is continuous from $A_{\gamma, \theta, p, T}^{h_{\gamma}}$ into $L^{2}(\Omega, \mathbb{P})$.

Eventually, thanks to Proposition 8.4, we can apply Proposition 8.10 to $a\left(s, s_{1}\right)=$ $f^{\prime}\left(W_{K}(s)\right)-f^{\prime}\left(W_{K}\left(s_{1}\right)\right)$ to get the continuity of

$$
K \rightarrow \int_{0}^{t} \mathrm{~d} B_{s} \int_{0}^{s} \Delta_{s, s_{1}}^{0} f^{\prime \prime}\left(W_{K}\right) I_{t}^{K}(K(\cdot, s))\left(s_{1}\right) \mathrm{d} B_{s_{1}}
$$

from $A_{\gamma, \theta, p, T}^{2 h_{\gamma}}$ into $L^{2}(\Omega, \mathbb{P})$.

\section{Appendix A. Pathwise versus stochastic integration}

We shall establish that for $W_{1}^{H}, W_{2}^{H}$ two independent fractional Brownian motions of index $0<H<\frac{1}{2}$,

$$
\int_{0}^{t} W_{1}^{H}(s) \mathrm{d} W_{2}^{H}(s)
$$

cannot be defined as a classical pathwise integral. To this end we recall that if $C^{\alpha}[0, T]$ denotes the space of Hölder continuous functions on $[0, T]$ of index $\alpha$ then:

$$
\begin{gathered}
\text { for every } \alpha \in(0, H) \text {, a.s } W^{H} \in C^{\alpha}[0, T], \\
\mathbb{P}\left(W^{H} \in C^{H}[0, T]\right)=0 .
\end{gathered}
$$

((A.1) can be proved with Kolmogorov's continuity criterion, and (A.2) is an immediate consequence of the law of the iterated logarithm, Theorem 1.7 of [2].) Let $v(f)$ be the 
variation index of the function $f$ :

$$
v(f)=\inf \left\{p>0: v_{p}(f)<+\infty\right\} .
$$

Then, see Theorem 5.3 of [11],

$$
\text { almost surely } \quad v\left(W^{H}\right)=\frac{1}{H} \text {. }
$$

(1) $\int_{0}^{t} W_{1}^{H}(s) \mathrm{d} W_{2}^{H}(s)$ cannot be defined as a $p$-variation integral. Indeed, see [11], this requires that for $p, q>0$ such that $\frac{1}{p}+\frac{1}{q}>1$,

$$
W_{1}^{H} \in \mathcal{W}_{p}([0, T]), \quad W_{2}^{H} \in \mathcal{W}_{q}([0, T]) .
$$

From (A.3) we deduce that $p, q \geqslant \frac{1}{H}$ and thus $H>\frac{1}{2}$.

(2) $\int_{0}^{t} W_{1}^{H}(s) \mathrm{d} W_{2}^{H}(s)$ cannot be defined as a generalized stochastic integral of Russo-Vallois [28] and Zähle [34]. Indeed, this integral requires that the integrand $W_{1}^{H}$ admits a generalized quadratic variation, and this implies by Proposition 5.1 [34], that $W_{1}^{H}$ is in the function space $\mathcal{W}_{2, \infty}^{\frac{1}{2}-}$. Since, see Theorem 1.1 [34], for $\varepsilon>0$ small enough

$$
\mathcal{W}_{2, \infty}^{\frac{1}{2}-\varepsilon} \subset I^{\frac{1}{2}-2 \varepsilon}\left(L_{2}\right) \subset C^{\frac{1}{2}-3 \varepsilon} .
$$

Hence, we need that $W_{1}^{H} \in C^{\frac{1}{2}-3 \varepsilon}$ for arbitrary small $\varepsilon$, and this implies $H \geqslant \frac{1}{2}$.

(3) $\int_{0}^{t} W_{1}^{H}(s) \mathrm{d} W_{2}^{H}(s)$ cannot be defined as a generalized integral of Ciesielski et al. [4]. If this were the case, then Section V.B implies that

$$
W_{1}^{H} \in \mathcal{B}_{p, 1}^{1-H} \quad \text { for } \frac{1}{p}<H<\frac{1}{p^{\prime}}=1-\frac{1}{p} .
$$

Since, for $\varepsilon>0$ small enough, $\mathcal{B}_{p, 1}^{1-H} \subset \mathcal{B}_{\infty, \infty}^{1-H-\varepsilon}=C^{1-H-\varepsilon}$, this implies again $H \geqslant \frac{1}{2}$.

\section{Appendix B. An elementary Calculus Lemma}

This lemma is used in Section 2 to evaluate the Hölder exponent of some integral processes.

LemmA B.1. - Let, for $0 \leqslant s \leqslant t \leqslant T$ and $\gamma \in(0,1) \cup(1,2)$,

$$
f(s, t)=\int_{0<u<s<v<t} \mathrm{~d} u \mathrm{~d} v(v-u)^{-\gamma} .
$$

Then,

$$
|f(s, t)| \leqslant C(T, \gamma)(t-s)^{h_{\gamma}} \quad(0 \leqslant s \leqslant t \leqslant T),
$$


with $h_{\gamma}=1$ if $\gamma \in(0,1)$ and $h_{\gamma}=2-\gamma$ if $\gamma \in(1,2)$. Similarly the function defined for $0 \leqslant s \leqslant t \leqslant T$ by

$$
g(s, t)=\int_{s<u<t<v<T}|u-v|^{-\gamma} \mathrm{d} u \mathrm{~d} v
$$

satisfies $|g(s, t)| \leqslant C|T-s|^{h_{\gamma}}$.

Proof. - Since $\gamma \notin\{1,2\}$, then

$$
f(s, t)=\frac{1}{(1-\gamma)(2-\gamma)}\left(t^{2-\gamma}-(t-s)^{2-\gamma}-s^{2-\gamma}\right) .
$$

If $1<\gamma<2$, this implies $|f(s, t)| \leqslant C(T, \gamma)(t-s)^{2-\gamma}$ since the function $x \rightarrow x^{\delta}$ is locally Hölder of index $\delta$ if $\delta \in(0,1)$.

If $0<\gamma<1$, then Taylor's formula yields $t^{2-\gamma}-s^{2-\gamma} \leqslant C(t, \gamma)(t-s)$ and this implies $|f(s, t)| \leqslant C(T, \gamma)(t-s)$.

\section{REFERENCES}

[1] E. Alos, O. Mazet, D. Nualart, Stochastic calculus with respect to fractional Brownian motion with Hurst parameter less than 1/2, Stochastic Process. Appl. 86 (2000) 121-139.

[2] A. Benassi, S. Jaffard, D. Roux, Elliptic Gaussian random processes, Rev. Mat. Iberoamericana 13 (1997) 19-90.

[3] J. Beran, N. Terrin, Testing for a change of the long-memory parameter, Biometrika 83 (1996) 627-638.

[4] Z. Ciesielski, G. Kerkyacharian, B. Roynette, Quelques espaces fonctionnels associés à des processus gaussiens. (Some function spaces associated with gaussian processes), Stud. Math. 107 (1993) 171-204.

[5] F. Comte, E. Renault, Long memory continuous time models, J. Econometrics 73 (1996) $101-150$.

[6] L. Coutin, Z. Qian, Stochastic analysis, rough path analysis and fractional Brownian motions, To be published in PTRF, 2000.

[7] L. Coutin, Z. Qian, Stochastic differential equations for fractional Brownian motions, C. R. Acad. Sci. Paris Sér. I Math. 331 (2000) 75-80.

[8] W. Dai, C. Heyde, Ito's formula with respect to fractional Brownian motion and its application, J. Appl. Math. Stochastic Anal. 9 (1996) 439-448.

[9] L. Decreusefond, A. Üstunel, Stochastic analysis of the fractional Brownian motion, Potential Anal. 10 (1997) 177-214.

[10] C. Dellacherie, B. Maisonneuve, P. Meyer, Probabilités et potentiel. Chapitres XVII à XXIV : Processus de Markov (fin) Compléments de calcul stochastique, Hermann, Paris, 1992.

[11] R. Dudley, R. Norvaisa, An introduction to $p$-variation and Young integrals, Tech. Rep. 1, Maphysto, Centre for Mathematical Physics and Stochastics, University of Aarhus, Ny Munkegade, DK-8000 Aarhus C, Denmark, 1998, Concentrated advanced course.

[12] T. Duncan, Y. Hu, B. Pasik-Duncan, Stochastic calculus for fractional Brownian motion I. Theory, SIAM J. Control Optim. 38 (2000) 582-612.

[13] D. Feyel, A. de La Pradelle, On the approximate solution of the Stratonovitch equation, Electron. J. Probab. 3 (1998). 
[14] A. Kolmogorov, Wienersche spiralen und einige andere interessante kurven im Hilbertschen raum, S. R. (Dokl.) Acad. Sci. USSR (N.S.) 26 (1940) 115-118.

[15] N. Lebedev, Special Functions and their Applications, Dover Publications, New York, 1972 (Translated and edited by Richard A. Silverman).

[16] W. Leland, M. Taqqu, W. Willinger, D. Wilson, On the self-similar nature of Ethernet traffic, IEEE/ACM Trans. Networking 2 (1994) 1-15.

[17] J. Leon, Fubini theorem for anticipating stochastic integrals in Hilbert space, Appl. Math. Optimization 27 (1993) 313-327.

[18] S. Lin, Stochastic analysis of fractional Brownian motions, Stochastics Stochastics Rep. 55 (1995) 121-140.

[19] R. Liptser, A. Shyriaev, Theory of Martingales, in: Mathematics and its Applications, Kluwer Academic Publishers, 1989.

[20] T. Lyons, Differential equations driven by rough signals, Rev. Mat. Iberoamericana 14 (1998) 215-310.

[21] I. Norros, E. Valkeila, J. Virtamo, An elementary approach to a Girsanov formula and other analytical results on fractional Brownian motions, Bernoulli 5 (1999) 571-588.

[22] D. Nualart, The Malliavin Calculus and Related Topics, in: Probability and its Applications, Springer-Verlag, New York, NY, 1995.

[23] V. Pipiras, M.S. Taqqu, Integration questions related to fractional Brownian motion, Probab. Theory Related Fields (2000) 251-291.

[24] N. Privault, Skorokhod stochastic integration with respect to non-adapted processes on Wiener space, Stochastics Stochastics Rep. 65 (1998) 13-39.

[25] P. Protter, Stochastic Integration and Differential Equations, in: Applications of Mathematics, Vol. 21, Springer-Verlag, 1992.

[26] L. Rogers, Arbitrage with fractional Brownian motion, Math. Finance 7 (1997) 95-105.

[27] F. Russo, P. Vallois, Forward, backward and symmetric stochastic integration, Probab. Theory Related Fields 97 (1993) 403-421.

[28] F. Russo, P. Vallois, The generalized covariation process and Itô formula, Stochastic Process. Appl. 59 (1995) 81-104.

[29] A.A. Ruzmaikina, Stieltjes integrals of Hölder continuous functions with applications to fractional Brownian motion, J. Statist. Phys. 100 (2000) 1049-1069.

[30] S. Samko, A. Kilbas, O. Marichev, Fractional Integrals and Derivatives, Gordon \& Breach Science, 1993.

[31] D. Stroock, A Concise Introduction to the Theory of Integration, 2nd edn., Birkhauser, 1994.

[32] L. Young, An inequality of Hölder type, connected with Stieltjes integration, Acta Math. 67 (1936) 251-282.

[33] M. Zähle, Integration with respect to fractal functions and Stochastic Calculus, Probab. Theory Related Fields 111 (1998) 333-374.

[34] M. Zähle, On the link between fractional and stochastic calculus, in: H. Crauel (Ed.), Stochastic Dynamics, Conference on Random Dynamical Systems, Bremen, Germany, April 28-May 2, 1997, Springer, 1999, pp. 305-325. Dedicated to Ludwig Arnold on the occasion of his 60th birthday. 